\title{
Analytical and numerical solutions of two-dimensional general rate models for liquid chromatographic columns packed with core-shell particles
}

\author{
Uche Ugochukwu David $^{\mathrm{a}, \mathrm{b}}$, Shamsul Qamar ${ }^{\mathrm{a}, \mathrm{c}, *}$, Andreas Seidel-Morgenstern ${ }^{\mathrm{c}}$ \\ ${ }^{a}$ Department of Mathematics, COMSATS Institute of Information Technology, \\ Park Road Chak Shahzad Islamabad, Pakistan \\ ${ }^{b}$ Department of Mathematics Programme, National Mathematical Centre Abuja, Nigeria \\ ${ }^{c}$ Max Planck Institute for Dynamics of Complex Technical Systems, Magdeburg, Germany
}

\begin{abstract}
This work is concerned with the analytical and numerical solutions of linear and nonlinear two-dimensional general rate models (2D-GRMs) describing the transport of single-solute and multi-component mixtures through chromatographic columns of cylindrical geometry packed with core-shell particles. The finite Hankel and Laplace transformations are successively applied to derive analytical solutions for a single-solute model considering linear adsorption isotherms and two different sets of boundary conditions. Moreover, analytical temporal moments are derived from the Laplace domain solutions. The process is further analyzed by numerically approximating the nonlinear 2D-GRM for core-shell particles considering multi-component mixtures and nonlinear Langmuir isotherm. A high resolution finite volume scheme is extended to solve the considered 2D-model equations. Several case studies of single-solute and multi-component mixtures are considered. The derived analytical results are validated against the numerical solutions of a high resolution finite volume scheme. Typical performance criteria are utilized to analyze the performance of the chromatographic process. The results obtained are considered to be useful to support further development of liquid chromatography.
\end{abstract}

Key words: Liquid chromatography, cylindrical column, two-dimensional general rate model, core-shell particles, analytical and numerical solutions, moment analysis.

\footnotetext{
${ }^{*}$ Corresponding author. Tel: +92-51-90495413; fax: +92-51-4442805 Email addresses: shamsul.qamar@comsats.edu.pk (Shamsul Qamar)
} 


\section{Introduction}

Chromatography exploits specific interactions of the components present in the mixtures to be separated with dedicated solid stationary phases. Numerous types of particles have been developed and are applied successfully [1-8]. Nonporous particles have been found successful in analytical liquid chromatography because they provide fast separation times [2-4]. The widely applied fully porous particles cause intraparticle mass transfer limitations reducing the column efficiencies. The use of core-shell particles (or superficially porous [1] or fusedcore [6] or cored beads [7]) can provide optimum conditions by avoiding the shortcomings of fully porous and nonporous particles. Fully porous particles can offer the large binding capacities with only moderate intraparticle mass transfer resistances [8]. The use of core-shell particles has generated recently considerable interests in both analytical and preparative liquid chromatography [9-11]. They have been used for example, for the separation of peptides and other compounds [10, 12], nucleotides [1], and proteins [13]. Moreover, several theoretical investigations have been carried out on the use of core-shell particles by considering onedimensional (1D) chromatographic models. Kaczmarski and Guichon [14] used the general rate model to study fully porous particles and the lumped particle model to study thin-shelled coated beads. Li et al. [15] carried out optimization of core size for linear chromatography by minimizing HETP numbers. Miyabe [5] showed that a column packed with cored beads can achieve higher resolution as compared to a column packed with fully-porous beads. Yang and $\mathrm{Hu}[16]$ derived the theoretical expressions of elution and frontal linear chromatography for ion-exchange resins that were cored beads. Wang et al. [7] studied the pressure-flow correlation with the ion-exchange resin of cored beads. They found that cored beads provided significantly enhanced rigidity and permeability compared to fully-porous homogeneous agarose beads [17]. $\mathrm{Gu}$ et al. [8] used the general rate model to study the optimization of the core radius fraction for multi-component isocratic elution with cored beads.

Analytical solutions and temporal moments of the 1D-models have been derived for linear isotherms using the Laplace transformation [18-23]. Moment analysis is a useful and effective technique for deducing important information about the retention equilibrium and mass transfer kinetics in a fixed-bed column. The moment generating property of the Laplace domain solutions can be used to derive analytical temporal moments. These moments can be used to get important information about the retention times, band broadenings, and front 
asymmetries. Several authors have derived moments for various boundary conditions (BCs) [18-37].

Recently, we have also derived analytical solutions and temporal moments of linear 2D-models for cylindrical columns packed with fully-porous particles [38-41]. Very recently, Qamar et al. $[22,42]$ have investigated linear and nonlinear 1D-models for core-shell particles. However, 2D-models have never been analyzed before for cylindrical columns packed with core-shell particles. This article extends the works carried out in $[22,42]$ for 1 D-models to linear and nonlinear 2D-GRMs considering core-shell particles.

For nonlinear adsorption equilibria the column model equations need to be solved numerically. The finite volume schemes have been widely applied to numerically approximate different chromatographic models and have been found suitable for simulating such nonlinear convection dominated problems [42-45]. These schemes were initially introduced for nonlinear hyperbolic equations. The slopes or flux limiters of these numerical schemes avoid numerical oscillations and over-predictions in the solutions and, thus, have capability to produce stable and accurate results [46].

In this article, analytical solutions and moments are derived for a linear single-solute 2D-GRM to study the effects of different kinetic parameters, especially the effects of radial dispersion on the elution profiles. Furthermore, the nonlinear multi-component 2D-GRM for core-shell particles is numerically approximated. A high resolution finite volume scheme, presented in $[42,43]$ for the 1D-models, is extended to solve the current 2D model equations.

$2 \mathrm{D}$ models can be valuable in various situations, e.g. a) the injection at the column inlet is not perfect (i.e. a radial profile is introduced at the column inlet), b) the column is not homogeneously packed (which is more and more probable the larger the columns are), c) there are radial temperature gradients, which are connected also with radial concentration gradients. All these issues occur in reality. Often they might be minor and even negligible, then justifying the 1D model. However, for evaluating the magnitude of the related effects 2D models are required. With already available isothermal models we could just study a special case for situation a) by assuming injections in inner cylinders or outer annuli. Situations b) and c) are more complicated and require further model extensions (considering non-constant column porosities and an energy balance), which are currently under investigation. The already developed simplified 2D-models and numerical scheme are more general and flexible 
compared to the classical 1D-models and numerical schemes [18-23, 43].

The remaining parts of this article are organized as follows. In Section 2, the 2D-GRM model is introduced. In Section 3, the analytical solutions of a linear single-solute 2D-GRM are derived for the considered two sets of boundary conditions. In Section 4, the analytical temporal moments are derived. Section 5 explains the proposed finite volume scheme applied to solve the nonlinear 2D-GRM. In Section 6, several case studies are presented. Lastly, concluding remarks are given in Section 7.

\section{The mathematical model of 2D-GRM}

In liquid chromatography, the GRM considers several contributions of the mass transfer processes that lead to band broadening. Mass transfer between the stationary and mobile phases, axial dispersion, and intraparticle pore diffusion are incorporated in the mass balance equations.

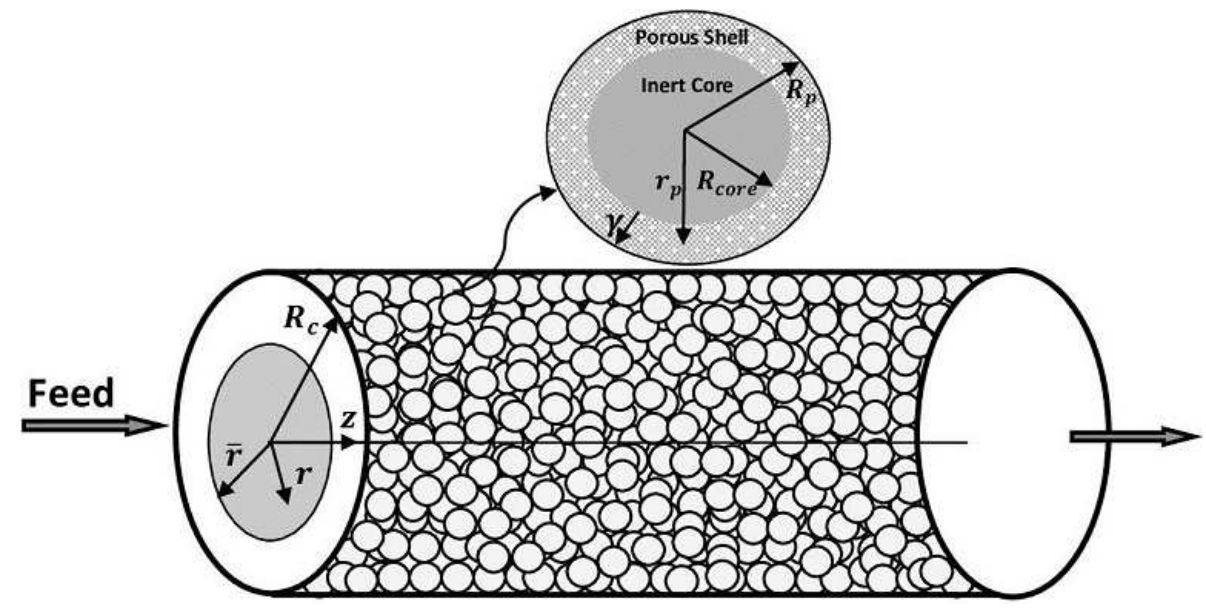

Figure 1: Schematic diagram of a chromatographic column of cylindrical geometry.

Let $t$ denotes the time coordinate, $z$ represents the axial coordinate along the column length, and $r$ is the radial coordinate along the column radius. The solute travels along the column axis in the $z$-direction by advection and axial dispersion and spreads along the column radius in the $r$-direction by radial dispersion. The following particular injection conditions are assumed to amplify the effects of mass transfer in the radial direction. A new parameter $\tilde{r}$ is introduced to split the inlet cross section of the column into an inner cylindrical core and an outer annular ring (see Figure 1). Thus, sample can be injected to the column either through 
an inner, through an outer ring, or through the whole cross section. The latter case results if $\tilde{r}$ is set equal to the radius of the column denoted by $R_{c}$. As no initial radial gradients are introduced in the latter case, the solutions should converge into the solution of simpler 1D-model [22].

In the above setup, both the axial and radial dispersions are considered. The mass balance equations in bulk of fluid for a multi-component mixture percolating through a cylindrical column packed with spherical particles are given as

$$
\frac{\partial c_{i}}{\partial t}+u \frac{\partial c_{i}}{\partial z}=D_{z, i} \frac{\partial^{2} c_{i}}{\partial z^{2}}+D_{r, i}\left(\frac{\partial^{2} c_{i}}{\partial r^{2}}+\frac{1}{r} \frac{\partial c_{i}}{\partial r}\right)-\frac{3}{R_{p}} F_{e} k_{\mathrm{ext}, i}\left(c_{i}-c_{p, i}\left(r_{p}=R_{p}\right)\right),
$$

where $i=1,2, \cdots, N_{c}$ and $N_{c}$ denotes the number of components in the mixtures. Here, $c_{i}$ is the concentration of $i$ th component in the bulk of fluid, $c_{p, i}$ is the concentration of the same component in the particle pores, $u$ is the interstitial velocity, $D_{z, i}$ is the axial dispersion coefficient of $i$ th component, and $F_{e}=(1-\epsilon) / \epsilon$ is the phase ratio with $\epsilon$ as the external porosity. Moreover, $D_{r, i}$ represents the radial dispersion coefficient and $k_{\text {ext }, i}$ is the external mass transfer coefficient of $i$ th component. Lastly, $r_{p}$ denotes the radial coordinate of spherical particles of radius $R_{p}$.

The corresponding mass balance equations inside the particles pores are given as

$$
\epsilon_{p} \frac{\partial c_{p, i}}{\partial t}+\left(1-\epsilon_{p}\right) \frac{\partial q_{p, i}}{\partial t}=\frac{\epsilon_{p} D_{p, i}}{r_{p}^{2}} \frac{\partial}{\partial r_{p}}\left(r_{p}^{2} \frac{\partial c_{p, i}}{\partial r_{p}}\right), i=1,2, \cdots, N_{c},
$$

where $q_{p, i}$ is the local equilibrium concentration of the solute in stationary phase for $i$ th component, $D_{p, i}$ is the pore diffusivity, and $\epsilon_{p}$ is the internal porosity.

To simplify the notations and reduce the number of variables, the following dimensionless quantities are introduced:

$$
\begin{aligned}
C_{i} & =\frac{c_{i}}{c_{\mathrm{inj}, i}}, \quad C_{p, i}=\frac{c_{p, i}}{c_{\mathrm{inj}, i}}, \quad q_{p, i}^{*}=\frac{q_{p, i}}{c_{\mathrm{inj}, i}}, \quad \tau=\frac{u t}{L}, \quad x=\frac{z}{L}, \quad \rho=\frac{r}{R_{c}}, \quad \rho_{p}=\frac{r_{p}}{R_{P}}, \\
P e_{z, i} & =\frac{L u}{D_{z, i}}, \quad P e_{\rho, i}=\frac{R_{c}^{2} u}{D_{r, i} L}, \quad \zeta_{i}=\frac{k_{\mathrm{ext}, i} R_{p}}{\epsilon_{p} D_{p, i}}, \quad \eta_{i}=\frac{\epsilon_{p} D_{p, i} L}{R_{p}^{2} u}, \quad \xi_{i}=3 \zeta_{i} \eta_{i} F_{e} .
\end{aligned}
$$

In the above equation, $c_{\mathrm{inj}, i}$ denotes the non-zero injected bulk concentration of $i$ th component, $P e_{z, i}$ is the Peclet number based on column length, $\zeta_{i}$ represents the modified Biot number, and $\eta_{i}$ describes the ratio of space time and interaparticle diffusion time for the $i$ th component. Using the above dimensionless variables, the model Eqs. (1) and (2) can be rewritten

$$
\frac{\partial C_{i}}{\partial \tau}+\frac{\partial C_{i}}{\partial x}=\frac{1}{P e_{z, i}} \frac{\partial^{2} C_{i}}{\partial x^{2}}+\frac{1}{P e_{\rho, i}}\left(\frac{\partial^{2} C_{i}}{\partial \rho^{2}}+\frac{1}{\rho} \frac{\partial C_{i}}{\partial \rho}\right)-\xi_{i}\left[C_{i}-C_{p, i}\left(\rho_{p}=1\right)\right],
$$




$$
\epsilon_{p} \frac{\partial C_{p, i}}{\partial \tau}+\left(1-\epsilon_{p}\right) \frac{\partial q_{p, i}^{*}}{\partial \tau}=\eta_{i}\left(\frac{\partial^{2} C_{p, i}}{\partial \rho_{p}^{2}}+\frac{1}{\rho_{p}} \frac{\partial C_{p, i}}{\partial \rho_{p}}\right), i=1,2, \cdots, N_{c} .
$$

In liquid chromatography, the frequently applied convex nonlinear Langmuir isotherm is defined as $[24,47]$

$$
q_{p, i}^{*}=\frac{a_{i} C_{p, i}}{1+\sum_{j=1}^{N_{c}}\left(b_{j} c_{\mathrm{inj}, j}\right) C_{p, j}}, \quad i=1,2, \cdots, N_{c}
$$

Here, $a_{i}$ denotes the Henry's constant and $b_{i}$ represents the extent of nonlinearly for component $i$. In the case of linear isotherms, $b_{i}=0$ for $i=1,2, \cdots, N_{c}$. Thus, Eq. (6) reduces to

$$
q_{p, i}^{*}=a_{i} C_{p, i}, \quad i=1,2, \cdots, N_{c} .
$$

For fully porous particles $\rho_{p}$ ranges from 0 to 1, while for core-shell particles it ranges from $\rho_{\text {core }}=R_{\text {core }} / R_{p}$ to 1 . As this study is concerned with the core-shell particles of arbitrary core radius fraction $\rho_{\text {core }}$, it is necessary to allow the core radius to be changed. For core-shell particles (c.f. Eq. (5)), $\rho_{\text {core }} \leq \rho_{p} \leq 1$. For fully porous particles $\rho_{\text {core }}=0$, while $\rho_{\text {core }} \neq 0$ for core-shell particles. Thus, following [8], it is helpful to replace $\rho_{p}$-axis by $0 \leq \gamma \leq 1$, where

$$
\gamma=\frac{\rho_{p}-\rho_{\text {core }}}{1-\rho_{\text {core }}}
$$

On substituting

$$
\rho_{p}=\gamma\left(1-\rho_{\text {core }}\right)+\rho_{\text {core }},
$$

in Eqs. (4) and (5) and using Eq. (6), they yield for $i=1,2, \cdots, N_{c}$

$$
\begin{gathered}
\frac{\partial C_{i}}{\partial \tau}+\frac{\partial C_{i}}{\partial x}=\frac{1}{P e_{z, i}} \frac{\partial^{2} C_{i}}{\partial x^{2}}+\frac{1}{P e_{\rho, i}}\left(\frac{\partial^{2} C_{i}}{\partial \rho^{2}}+\frac{1}{\rho} \frac{\partial C_{i}}{\partial \rho}\right)-\xi_{i}\left(C_{i}-\left.C_{p, i}\right|_{\gamma=1}\right), \\
\epsilon_{p} \frac{\partial C_{p, i}}{\partial \tau}+\left(1-\epsilon_{p}\right) \frac{\partial q_{p, i}^{*}}{\partial \tau}=\eta_{i}\left[\frac{1}{\left(1-\rho_{\text {core }}\right)^{2}} \frac{\partial^{2} C_{p, i}}{\partial \gamma^{2}}+\frac{2}{\gamma\left(1-\rho_{\text {core }}\right)^{2}+\rho_{\text {core }}\left(1-\rho_{\text {core }}\right)} \frac{\partial C_{p, i}}{\partial \gamma}\right] .
\end{gathered}
$$

The Eqs. (10) and (11) are subjected to the initial and boundary conditions. The initial conditions for an initially regenerated column are given as

$$
C_{i}(x, \rho, \tau=0)=0, \quad C_{p, i}(x, \rho, \gamma, \tau=0)=0, \quad \forall x, \rho, \gamma \in(0,1), i=1,2, \cdots, N_{c} .
$$


The following boundary conditions for Eq. (10) are used along the column radius at $\rho=0$ and $\rho=1$

$$
\frac{\partial C_{i}(x, \rho=0, \tau)}{\partial \rho}=0, \quad \frac{\partial C_{i}(x, \rho=1, \tau)}{\partial \rho}=0 .
$$

The first and second boundary conditions corresponds to the symmetry of radial profile and the impermeability of the column wall, respectively. The following boundary conditions at $\gamma=0$ and $\gamma=1$ are assumed for Eq. (11)

$$
\left.\frac{\partial C_{p, i}}{\partial \gamma}\right|_{\gamma=0}=0,\left.\quad \frac{\partial C_{p, i}}{\partial \gamma}\right|_{\gamma=1}=\left(1-\rho_{\text {core }}\right) \zeta_{i}\left(C_{i}-\left.C_{p, i}\right|_{\gamma=1}\right) .
$$

Two sets of boundary conditions are considered at the column inlet and outlet as summarized below:

Case 1: Concentration pulse of finite width is injected as Dirichlet inlet BCs:

For injection in the inner circular region, it is expressed as:

$$
C_{i}(x=0, \rho, \tau)=\left\{\begin{array}{cc}
1, & \text { if } 0 \leq \rho \leq \tilde{\rho} \text { and } 0 \leq \tau \leq \tau_{\text {inj }}, \\
0, & \text { if } \tilde{\rho}<\rho \leq 1 \text { or } \tau>\tau_{\text {inj }}
\end{array}\right.
$$

For injection in the outer annular zone, it is expressed as:

$$
C_{i}(x=0, \rho, \tau)=\left\{\begin{array}{cc}
1, & \text { if } \tilde{\rho}<\rho \leq 1 \text { and } 0 \leq \tau \leq \tau_{\text {inj }} \\
0, & \text { if } 0 \leq \rho \leq \tilde{\rho} \text { or } \tau>\tau_{\text {inj }}
\end{array}\right.
$$

and

$$
\tilde{\rho}=\tilde{r} / R_{c} .
$$

For injection over the whole inlet cross section of the column, either $\tilde{\rho}=1$ in Eq. (15) or $\tilde{\rho}=0$ in Eq. (16). In the case of more complicated boundary conditions, the analytical solutions may not be possible. However, the proposed numerical scheme is able to handle more general boundary conditions.

At the outlet of the column of hypothetically infinite length, $x=\infty$, the following outflow Neumann boundary condition is considered:

$$
\left.\frac{\partial C_{i}(x, \rho, \tau)}{\partial x}\right|_{x=\infty}=0
$$

Case 2: Concentration pulse of finite width injected as Danckwerts inlet BCs : 
For the inner zone injection, the boundary condition is expressed as:

$$
C_{i}(x=0, \rho, \tau)-\frac{1}{P e_{z, i}} \frac{\partial C_{i}(x=0, \rho, \tau)}{\partial x}=\left\{\begin{array}{lc}
1, & \text { if } 0 \leq \rho \leq \tilde{\rho} \text { and } 0 \leq \tau \leq \tau_{\text {inj }} \\
0, & \tilde{\rho}<\rho \leq 1 \text { or } \tau>\tau_{\text {inj }}
\end{array}\right.
$$

For the injection through outer annular zone, it is expressed as:

$$
C_{i}(x=0, \rho, \tau)-\frac{1}{P e_{z, i}} \frac{\partial C_{i}(x=0, \rho, \tau)}{\partial x}=\left\{\begin{array}{l}
1, \quad \text { if } \tilde{\rho}<\rho \leq 1 \text { and } 0 \leq \tau \leq \tau_{\text {inj }} \\
0, \quad 0 \leq \rho \leq \tilde{\rho} \text { or } \tau>\tau_{\text {inj }}
\end{array}\right.
$$

together with the Neumann condition at the outlet of a finite length column

$$
\left.\frac{\partial C_{i}(x, \rho, \tau)}{\partial x}\right|_{x=1}=0
$$

The Danckwerts boundary condition tends to the Dirichlet boundary condition for large values of $P e_{z, i}$.

\section{Derivation of analytical solutions for linear single-solute model}

Here, we consider the flow of single-solute $\left(N_{c}=1\right)$ and linear adsorption isotherm (c.f. Eq. (7)) for deriving analytical solutions and moments. This assumption of linear chromatography is valid for diluted sample. After using Eq. (7) in Eqs. (10) and (11) and considering $N_{c}=1$, $C_{1}=C, C_{p, 1}=C_{p}$, we obtain

$$
\begin{aligned}
& \frac{\partial C}{\partial \tau}+\frac{\partial C}{\partial x}=\frac{1}{P e_{z}} \frac{\partial^{2} C}{\partial x^{2}}+\frac{1}{P e_{\rho}}\left(\frac{\partial^{2} C}{\partial \rho^{2}}+\frac{1}{\rho} \frac{\partial C}{\partial \rho}\right)-\xi\left(C-\left.C_{p}\right|_{\gamma=1}\right), \\
& \left(1-\rho_{\text {core }}\right)^{2} a^{*} \frac{\partial C_{p}}{\partial \tau}-\eta\left(\frac{\partial^{2} C_{p}}{\partial \gamma^{2}}+\frac{2\left(1-\rho_{\text {core }}\right)}{\left(\gamma\left(1-\rho_{\text {core }}\right)+\rho_{\text {core }}\right)} \frac{\partial C_{p}}{\partial \gamma}\right)=0,
\end{aligned}
$$

where

$$
a^{*}=\epsilon_{p}+a\left(1-\epsilon_{p}\right)
$$

The above chromatographic model and its associated initial and boundary conditions are an-

alytically solved by successive implementation of the finite Hankel transform and the Laplace 
transform. The zeroth-order finite Hankel transform of $C(x, \rho, \tau)$ is defined below as (c.f. $[48-51])$

$$
C_{H}\left(x, \lambda_{n}, \tau\right)=H[C(x, \rho, \tau)]=\int_{0}^{1} C(x, \rho, \tau) J_{0}\left(\lambda_{n} \rho\right) \rho d \rho .
$$

The inverse Hankel transform is given as

$$
C(x, \rho, \tau)=2 C_{H}\left(x, \lambda_{n}=0, \tau\right)+2 \sum_{n=1}^{\infty} C_{H}\left(x, \lambda_{n}, \tau\right) \frac{J_{0}\left(\lambda_{n} \rho\right)}{\left|J_{0}\left(\lambda_{n}\right)\right|^{2}} .
$$

Eq. (23) is rephrased as

$$
\left(1-\rho_{\text {core }}\right)^{2} a^{*} \frac{\partial}{\partial \tau}\left[\left(\gamma\left(1-\rho_{\text {core }}\right)+\rho_{\text {core }}\right) C_{p}\right]-\eta \frac{\partial^{2}}{\partial \gamma^{2}}\left(\left(\gamma\left(1-\rho_{\text {core }}\right)+\rho_{\text {core }}\right) C_{p}\right)=0,
$$

with (c.f. Eq. (14))

$$
\left.\frac{\partial C_{p}}{\partial \gamma}\right|_{\gamma=0}=0,\left.\quad \frac{\partial C_{p}}{\partial \gamma}\right|_{\gamma=1}=\left(1-\rho_{\text {core }}\right) \zeta\left(C-\left.C_{p}\right|_{\gamma=1}\right) .
$$

The remaining solution procedure is similar to one presented in our previous article [41] and is, therefore, omitted here. Thus, the general Hankel-Laplace domains solution is given as

$$
\bar{C}_{H}\left(x, \lambda_{n}, s\right)=A_{0} e^{m_{1} x}+B_{0} e^{m_{2} x}
$$

where

$$
m_{1,2}=\frac{P e_{z}}{2}\left(1 \pm \sqrt{1+\frac{4 \phi\left(s, \lambda_{n}\right)}{P e_{z}}}\right) .
$$

In Eq. (30), $m_{1}$ is obtained by using the plus sign and $m_{2}$ is extracted using the minus sign. The next step is to find the integration constants $A_{0}$ and $B_{0}$ by using the considered two types of boundary conditions.

Case 1: Concentration pulse of finite width is injected as Dirichlet inlet BCs:

The Hankel transformations of Eqs. (15) (or Eqs. (16)) and (18) are given as

$$
\begin{gathered}
C_{H}\left(x, \lambda_{n}, \tau\right)=\left\{\begin{array}{lc}
F\left(\lambda_{n}\right), & \text { if } 0 \leq \tau \leq \tau_{\mathrm{inj}}, \\
0, & \text { if } \tau>\tau_{\mathrm{inj}},
\end{array}\right. \\
\left.\frac{\partial C_{H}\left(x, \lambda_{n}, \tau\right)}{\partial x}\right|_{x=\infty}=0 .
\end{gathered}
$$

For injection at the inner cylindrical core, $F\left(\lambda_{n}\right)$ is given as

$$
F\left(\lambda_{n}\right)=\left\{\begin{array}{lr}
\frac{\tilde{\rho}^{2}}{2}, & \text { if } \lambda_{n}=0 \\
\frac{\tilde{\rho}}{\lambda_{n}} J_{1}\left(\lambda_{n} \tilde{\rho}\right), & \text { if } \lambda_{n} \neq 0
\end{array}\right.
$$


while for injection at the outer annular ring, it becomes

$$
F\left(\lambda_{n}\right)= \begin{cases}\left(\frac{1}{2}-\frac{\tilde{\rho}^{2}}{2}\right), & \text { if } \lambda_{n}=0 \\ -\frac{\tilde{\rho}}{\lambda_{n}} J_{1}\left(\lambda_{n} \tilde{\rho}\right), & \text { if } \lambda_{n} \neq 0\end{cases}
$$

After applying the Laplace transformation on boundary conditions in Eqs. (31) and (32), we obtain

$$
\bar{C}_{H}\left(x, \lambda_{n}, s\right)=\frac{F\left(\lambda_{n}\right)}{s}\left(1-e^{-s \tau_{\mathrm{inj}}}\right),\left.\quad \frac{\partial \bar{C}_{H}}{\partial x}\right|_{x=\infty}=0 .
$$

Now, using Eq. (35) in Eq. (29), we obtain the following solution for the Dirichlet boundary conditions

$$
\bar{C}_{H}\left(x, \lambda_{n}, s\right)=\frac{\left(1-e^{-s \tau_{\text {inj }}}\right)}{s} F\left(\lambda_{n}\right) e^{m_{2} x},
$$

where $m_{2}$ is given by Eq. (30) for the lower negative sign.

Case 2: Concentration pulse of finite width injected as Danckwerts inlet BCs :

The Hankel transformations of Eqs. (19) (or Eqs. (20)) and (21) are given as

$$
C_{H}\left(x=0, \lambda_{n}, \tau\right)-\frac{1}{P e_{z}} \frac{\partial C_{H}\left(x=0, \lambda_{n}, \tau\right)}{\partial x}=\left\{\begin{array}{lc}
F\left(\lambda_{n}\right), & \text { if } 0 \leq \tau \leq \tau_{\mathrm{inj}} \\
0, & \text { if } \tau>\tau_{\mathrm{inj}}
\end{array}\right.
$$

together with the Neumann condition at the outlet of the column

$$
\left.\frac{\partial C_{H}\left(x, \lambda_{n}, \tau\right)}{\partial x}\right|_{x=1}=0
$$

Here, $F\left(\lambda_{n}\right)$ is given by Eq. (33) for the inner injection and by Eq. (34) for the outer annular injection.

After applying the Laplace transformation on these boundary conditions, we get

$$
\bar{C}_{H}\left(x=0, \lambda_{n}, s\right)-\frac{1}{P e_{z}} \frac{\partial \bar{C}_{H}\left(x=0, \lambda_{n}, s\right)}{\partial x}=\frac{F\left(\lambda_{n}\right)}{s}\left(1-e^{-s \tau_{\mathrm{inj}}}\right),
$$

and

$$
\left.\frac{\partial \bar{C}_{H}}{\partial x}\right|_{x=1}=0 .
$$

On using Eqs. (39) and (40) in Eq. (29), we obtain the following solution for the Danckwerts boundary conditions

$$
\bar{C}_{H}\left(x, \lambda_{n}, s\right)=\frac{\left[m_{2} e^{m 2+m_{1} x}-m_{1} e^{m 1+m_{2} x}\right]\left[\frac{F\left(\lambda_{n}\right)}{s}\left(1-e^{-s \tau_{\mathrm{inj}}}\right)\right]}{m_{2} e^{m_{2}}\left(1-\frac{m_{1}}{P e_{z}}\right)-m_{1} e^{m_{1}}\left(1-\frac{m_{2}}{P e_{z}}\right)} .
$$


Analytical Laplace inversion is not possible for the solutions of both the Dirichlet and Danckwerts boundary conditions. Thus, the numerical Laplace inversion will be applied to get semi-analytical solutions in the actual time domain $[52,53]$. In this technique, the integral term appearing in the inverse Laplace transform is approximated by Fourier series, see [52] for more details.

\section{Moment Analysis}

Moment analysis is a well known useful technique for collecting relevant information about the retention equilibrium and mass transfer kinetics in a column. A set of statistical temporal moments can be used to describe the appearance of elution profile. For instance, the appropriate forms of first, second, third and fourth moments can describe the mean, spread, skewness, and kurtosis of the elution profiles, respectively. The experimental values measured for these moments can be compared with their theoretical expressions to estimate mass transfer coefficients. If there are baseline shifts, specific measures are required to take them into account.

The normalized $i$-th moment averaged over the radial coordinate $(\rho)$ of the band profile at any position in the column can be obtained through the following expression

$$
\mu_{0, \mathrm{av}}=\int_{0}^{\infty} C_{\mathrm{av}}(x, \tau) d \tau, \quad \mu_{i, \mathrm{av}}=\frac{\int_{0}^{\infty} C_{\mathrm{av}}(x, \tau) \tau^{i} d \tau}{\mu_{0, \mathrm{av}}}, \quad i=1,2,3, \cdots,
$$

where

$$
C_{\mathrm{av}}(x, \tau)=2 \int_{0}^{1} C(x, \rho, \tau) \rho d \rho .
$$

Due to its moment generating property, the Laplace transformation can be used to obtain analytical expressions for the moments. Temporal moments are derived analytically as functions of radial coordinate $\rho$ at the outlet of the column $(x=1)$. The following relation is utilized to obtain analytical temporal moments from the Hankel and Laplace transformed concentration $\bar{C}_{H}$ in Eq. (36) or (41)

$$
\mu_{i, H}=(-1)^{i} \lim _{s \rightarrow 0} \frac{d^{i}\left(\bar{C}_{H}\left(x, \lambda_{n}, s\right)\right)}{d s^{i}}, \quad i=0,1,2, \cdots .
$$

The true moments $\mu_{i}(\rho)$ are obtained from Eq. (26) by taking the $i$-th moment of concentration on the both sides of that equation. Thus, on multiplying both sides of Eq. (26) with $\tau^{i}$ 
and integrating over $\tau$ from 0 to $\infty$, we get

$$
\mu_{i}(\rho)=2 \mu_{i, H}\left(\lambda_{n}=0\right)+2 \sum_{n=1}^{\infty} \mu_{i, H}\left(\lambda_{n}\right) \frac{J_{0}\left(\lambda_{n} \rho\right)}{\left|J_{0}\left(\lambda_{n}\right)\right|^{2}} .
$$

Further, the averaged non-normalized temporal moments $M_{i, \text { av }}$ are determined as

$$
M_{i, \mathrm{av}}=2 \int_{0}^{1} \mu_{i}(\rho) \rho d \rho, \quad i=0,1,2, \cdots .
$$

Lastly, the normalized averaged temporal moments, defined in Eq. (42) and frequently used in chemical engineering [24], are given as

$$
\mu_{i, \mathrm{av}}=\frac{M_{i, \mathrm{av}}}{\mu_{0, \mathrm{av}}}, \quad \mu_{0, \mathrm{av}}=M_{0, \mathrm{av}}, \quad i=1,2,3, \cdots .
$$

The above temporal moments $\mu_{i \text {,av }}$ up to fourth order are obtained to interpret the behavior of a solute moving through the column. The first three central moments can be obtained as $[24]$

$$
\begin{aligned}
& \mu_{2, \mathrm{av}}^{\prime}=\mu_{2, \mathrm{av}}-\mu_{1, \mathrm{av}}^{2}, \\
& \mu_{3, \mathrm{av}}^{\prime}=\mu_{3, \mathrm{av}}-3 \mu_{1, \mathrm{av}} \mu_{2, \mathrm{av}}+2 \mu_{1, \mathrm{av}}^{3}, \\
& \mu_{4, \mathrm{av}}^{\prime}=\mu_{4, \mathrm{av}}-4 \mu_{1, \mathrm{av}} \mu_{3, \mathrm{av}}+6 \mu_{1, \mathrm{av}}^{2} \mu_{2, \mathrm{av}}-3 \mu_{1, \mathrm{av}}^{4} .
\end{aligned}
$$

The corresponding numerical $i$-th moment of the band profile at the outlet of the column of length $x=1$ is obtained as

$$
\mu_{i, \mathrm{av}}=\frac{\int_{0}^{\infty} C_{\mathrm{av}}(x=1, \tau) \tau^{i} d \tau}{\mu_{0, \mathrm{av}}}, \quad i=2,3,4, \cdots
$$

where, $\mu_{0, \text { av }}$ for $x=1$ is given by Eq. (42). The trapezoidal rule is utilized to approximate the integrals appearing in Eqs. (42) and (51).

The column efficiency $N$, which is also known as the plate number, and the height of an equivalent theoretical plate (HETP) can be calculated using the moments [15, 24]. This is a well known common measure for ideal mass transfer within packed beds in chemical engineering and is calculated for negligible pulse width as:

$$
N=\frac{\mu_{1, \mathrm{av}}^{2}}{\mu_{2, \mathrm{av}}^{\prime}}, \quad \operatorname{HETP}=\frac{1}{N},
$$

where $\mu_{1, \text { av }}^{2}$ is the square of the first averaged moment and $\mu_{2, \text { av }}^{\prime}$ is the second central averaged moment.

The analytical moments of 2D-GRM for the considered two sets of boundary conditions are presented in Appendix A. 


\section{Numerical Scheme}

Various numerical schemes have been applied in the literature to approximate 1D chromatographic models of different complexities [20, 24, 43, 54]. In this work, a semi-discrete high resolution flux-limiting finite volume scheme, presented in $[20,43,54]$ for the $1 \mathrm{D}$ models, is extended to approximate the current 2D-model solutions. A second order total variation diminishing Rung-Kutta (TVD-RK) method of order 2 is applied to solve the resulting system of ordinary differential equations [55]. In our previous article [43], we have proved analytically and numerically that this scheme is second order accurate.

For the sake of simplicity and better understanding, we derive the scheme for the case of onecomponent 2D-GRM only. Extension of this scheme to two and three-component mixtures is straightforward.

In order to derive this scheme, let $N_{x}, N_{\rho}$ and $N_{\gamma}$ be the large integers in $x, \rho$ and $\gamma$ coordinates respectively. We consider a domain $[0,1] \times[0,1] \times[0,1]$ which is covered by cells $\Omega_{k l m} \equiv\left[x_{k-\frac{1}{2}}, x_{k+\frac{1}{2}}\right] \times\left[\rho_{l-\frac{1}{2}}, \rho_{l+\frac{1}{2}}\right] \times\left[\gamma_{m-\frac{1}{2}}, \gamma_{m+\frac{1}{2}}\right]$ for $1 \leq k \leq N_{x}, 1 \leq l \leq N_{\rho}$ and $1 \leq m \leq N_{\gamma}$. The representative coordinate points in the cell $\Omega_{k l m}$ are denoted by $\left(x_{k}, \rho_{l}, \gamma_{m}\right)$.

Here,

$$
x_{k}=\frac{x_{k-\frac{1}{2}}+x_{k+\frac{1}{2}}}{2}, \quad \rho_{l}=\frac{\rho_{l-\frac{1}{2}}+\rho_{l+\frac{1}{2}}}{2}, \quad \gamma_{m}=\frac{\gamma_{m-\frac{1}{2}}+\gamma_{m+\frac{1}{2}}}{2}
$$

and for the current uniform mesh

$$
\Delta x=x_{k-\frac{1}{2}}-x_{k+\frac{1}{2}}, \quad \Delta \rho=\rho_{l-\frac{1}{2}}-\rho_{l+\frac{1}{2}}, \quad \Delta \gamma=\gamma_{m-\frac{1}{2}}-\gamma_{m+\frac{1}{2}}
$$

Note that

$$
C:=C(x, \rho, t) \quad \text { and } \quad C_{p}:=C_{p}(x, \rho, \gamma, t)
$$

Therefore, for $I_{k l}:=\left[x_{k-\frac{1}{2}}, x_{k+\frac{1}{2}}\right] \times\left[\rho_{l-\frac{1}{2}}, \rho_{l+\frac{1}{2}}\right]$ and $\Omega_{k l m}:=\left[x_{k-\frac{1}{2}}, x_{k+\frac{1}{2}}\right] \times\left[\rho_{l-\frac{1}{2}}, \rho_{l+\frac{1}{2}}\right] \times$ $\left[\gamma_{m-\frac{1}{2}}, \gamma_{m+\frac{1}{2}}\right]$, the cell averaged values $C_{k, l}(t)$ and $C_{p, k, l, m}(t)$ at any time $t$ are expressed as

$$
\begin{aligned}
& C_{k, l}=C_{k, l}(t)=\frac{1}{\Delta x_{k} \Delta \rho_{l}} \int_{I_{k}} C(\rho, x, t) d \rho d x \\
& C_{p, k, l, m}=C_{p, k, l, m}(t)=\frac{1}{\Delta x_{k} \Delta \rho_{l} \Delta \gamma_{m}} \int_{\Omega_{k l m}} C(\gamma, \rho, x, t) d \gamma d \rho d x
\end{aligned}
$$


On integrating Eq. (10) for $N_{c}=1$ over the interval $I_{k l}$ and using Eqs. (56) and (57), we obtain

$$
\begin{aligned}
\frac{d C_{k, l}}{d \tau}= & -\frac{C_{k+\frac{1}{2}, l}-C_{k-\frac{1}{2}, l}}{\Delta x}+\frac{1}{\Delta x P e_{z}}\left[\left(\frac{\partial C}{\partial x}\right)_{k+\frac{1}{2}, l}-\left(\frac{\partial C}{\partial x}\right)_{k-\frac{1}{2}, l}\right]-\xi\left(C_{k, l}-C_{p, k, l, N_{\gamma}}\right) \\
& +\frac{1}{\Delta \rho P e_{\rho}}\left[\left(\frac{\partial C}{\partial \rho}\right)_{k, l+\frac{1}{2}}-\left(\frac{\partial C}{\partial \rho}\right)_{k, l-\frac{1}{2}}+\frac{C_{k, l+\frac{1}{2}}-C_{k, l-\frac{1}{2}}}{\rho_{l+\frac{1}{2}}}\right]
\end{aligned}
$$

where $k=1,2, \ldots, N_{x}$ and $l=1,2, \ldots, N_{\rho}$. The differential terms of the axial and radial diffusion parts can be approximated as:

$$
\left(\frac{\partial C}{\partial x}\right)_{k \pm \frac{1}{2}, l}= \pm \frac{\left(C_{k, \pm 1, l}-C_{k, l}\right)}{\Delta x}
$$

and

$$
\left(\frac{\partial C}{\partial \rho}\right)_{k, l \pm \frac{1}{2}}= \pm \frac{\left(C_{k, l \pm 1}-C_{k, l}\right)}{\Delta \rho}
$$

Moreover, integration of Eq. (11) over the interval $\Omega_{k l m}$ gives

$$
\frac{d C_{p, k, l, m}}{d \tau}=\frac{\eta}{J_{k, l, m}\left(1-\rho_{\text {core }}\right)^{2} \Delta \gamma}\left[\left(\frac{\partial C_{p}}{\partial \gamma}\right)_{k, l, m+\frac{1}{2}}-\left(\frac{\partial C_{p}}{\partial \gamma}\right)_{k, l, m-\frac{1}{2}}+\frac{2\left(C_{p, k, l, m+\frac{1}{2}}-C_{p, k, l, m-\frac{1}{2}}\right)}{\gamma_{m+\frac{1}{2}}+\frac{\rho_{\text {core }}}{\left(1-\rho_{\text {core }}\right)}}\right],
$$

where due to Langmuir isotherm in Eq. (6)

$$
J_{k, l, m}=\epsilon_{p}+\frac{\left(1-\epsilon_{p}\right) a}{\left(1+\left(b c_{\text {inj }}\right) C_{p, k, l, m}\right)^{2}}
$$

and

$$
\left(\frac{\partial C_{p}}{\partial \gamma}\right)_{k, l, m \pm \frac{1}{2}}= \pm \frac{\left(C_{k, l, m \pm 1}-C_{k, l, m}\right)}{\Delta \gamma}
$$

The next step is to device a procedure for approximating concentration fluxes at the cell interfaces $x_{k \pm \frac{1}{2}}, \rho_{l \pm \frac{1}{2}}$ and $\gamma_{m \pm \frac{1}{2}}$ in Eqs. (58) and (61). Several numerical schemes can be used for approximating these fluxes. In this study, the following first and second order accurate methods are used to approximate concentrations at the cell interfaces, while the TVD-RK scheme is applied to obtain a second order accuracy in time [54, 55].

Since $\frac{\eta}{J_{k, l, m}\left(1-\rho_{\text {core }}\right)^{2} \Delta \gamma}$ and the velocity $u$ are both positive, the contractions $C$ and $C_{p}$ at the cell interfaces are approximated in the following manner

$$
C_{k, l+\frac{1}{2}}=C_{k, l} \quad C_{k, l-\frac{1}{2}}=C_{k, l-1},
$$




$$
C_{p, k, l, m+\frac{1}{2}}=C_{p, k, l, m} \quad C_{p, k, l, m-\frac{1}{2}}=C_{p, k, l, m-1}
$$

Eqs. (64) and (65) give a first order accuracy of the numerical scheme in the axial- and radial-coordinates. To obtain second order accurate scheme, the fluxes are approximated as

$$
\begin{gathered}
C_{k, l+\frac{1}{2}}=C_{k, l}+\frac{1}{2} \varphi\left(\mu_{k, l}\right)\left(C_{k, l}-C_{k, l-1}\right), \quad \mu_{k, l}=\frac{C_{k, l+1}-C_{k, l}+\xi}{C_{k, l}-C_{k, l-1}+\xi}, \\
C_{p, k, l, m+\frac{1}{2}}=C_{p, k, l, m}+\frac{1}{2} \phi\left(\nu_{k, l, m}\right)\left(C_{p, k, l, m}-C_{p, k, l, m-1}\right), \quad \nu_{k, l, m}=\frac{C_{p, k, l, m+1}-C_{p, k, l, m}+\xi}{C_{p, k, l, m}-C_{p, k, l, m-1}+\xi} .
\end{gathered}
$$

Eqs. (66) and (67) produce a flux-limiting high resolution scheme. Here, a small number $\xi=10^{-10}$ is used to prevent division by zero. The flux limiting functions $\varphi$ and $\phi$ are used to preserve the local monotonicity (positivity) of the scheme as defined below $[43,54]$

$$
\begin{aligned}
\varphi\left(\mu_{k, l}\right) & =\max \left(0, \min \left(2 \mu_{k, l}, \min \left(\frac{1}{3}+\frac{2}{3} \mu_{k, l}, 2\right)\right)\right), \\
\phi\left(\nu_{k, l, m}\right) & =\max \left(0, \min \left(2 \nu_{k, l, m}, \min \left(\frac{1}{3}+\frac{2}{3} \nu_{k, l, m}, 2\right)\right)\right) .
\end{aligned}
$$

At the boundary intervals, the proposed high resolution scheme is not applicable. Thus, the first order backward approximation is applied at the cell boundary fluxes, while the high resolution scheme is used to compute the fluxes at all interior cell interfaces. It is should be noted that the use of first order scheme will not diminish the global accuracy of the proposed numerical scheme, see [54] for more details.

Finally, a second order TVD-RK scheme is used to solve Eqs.(58)-(69) to obtain the second order accuracy in time [55]. The right-hand-side of Eqs. (58) and (61) are denoted as $\mathcal{L}\left(C,\left.C_{p}\right|_{\gamma=1}\right)$ and $\mathcal{M}\left(C_{p}\right)$. The following stages are used to update $C$ and $C_{p}$ by a second order TVD Runge-Kutta scheme [55]:

$$
\begin{gathered}
C^{(1)}=C^{n}+\Delta \tau \mathcal{L}\left(C^{n},\left.C_{p}^{n}\right|_{\gamma=1}\right), \quad C_{p}^{(1)}=C_{p}^{n}+\Delta \tau \mathcal{M}\left(C_{p}^{n}\right), \\
C^{n+1}=\frac{1}{2}\left[C^{n}+C^{(1)}+\Delta \tau \mathcal{L}\left(C^{(1)},\left.C_{p}^{(1)}\right|_{\gamma=1}\right)\right], \quad C_{p}^{n+1}=\frac{1}{2}\left[C_{p}^{n}+C_{p}^{(1)}+\Delta \tau \mathcal{M}\left(C_{p}^{(1)}\right)\right],
\end{gathered}
$$

where $C$ and $C_{p}$ are solutions at the previous time step $\tau^{n}$ and $C^{n+1}$ and $C_{p}^{n+1}$ are updated solutions at the next time step $\tau^{n+1}$. Here, the time step $\Delta \tau$ is calculated under the following Courant-Friedrichs-Lewy (CFL) condition:

$$
\Delta \tau \leq \frac{1}{2} \min \left(\Delta x, \Delta x^{2} \min \left(P e_{z, i}\right), \frac{\Delta \gamma^{2}\left(1-\rho_{\text {core }}\right)^{2}}{\max \left(J_{k, l, m}^{-1} \eta\right)}, \frac{\rho_{\text {core }}\left(1-\rho_{\text {core }}\right) \Delta \gamma}{2 \max \left(J_{k, l, m}^{-1} \eta\right)}\right) .
$$


The above numerical scheme was programmed in $\mathrm{C}$ language with $50 \times 20 \times 20$ grid points and was executed on a laptop computer containing dual-core Intel processor with a random access memory of 8 gigabytes.

\subsection{Process performance criteria}

Industrial processes need optimization for efficiency, productivity and purity, the same is also true for preparative chromatographic processes. A performance criterion needed for the assessment of quality of a product was introduced in [56]. The same criteria is utilized in this work to assess the performance of a cylindrical chromatographic column packed with core-shell particles. A two component mixture is considered in which component 1 has lower affinity for the stationary phase as compared to the component 2 , i.e. $a_{1}<a_{2}$. Let $c_{i}=c_{\mathrm{inj}, i} C_{i}$ denote the concentration of $i$ th component in grams per liter, where $C_{i}$ is the dimensionless concentration normalized with $c_{\text {inj }, i}$ as defined in Eq. (3). Let $\tau_{1}$ be the dimensionless time at which the fraction of component 1 exceeds some threshold, i.e. $c_{1} \geq \epsilon c_{\text {inj, }, 1}$, where $\epsilon=10^{-5}$

in this case. Moreover, let $\tau_{2}$ be the time at which the fraction of component 2 drops below some threshold $c_{2} \leq \epsilon c_{\text {inj,1 }}$. The cycle time is the time lapse between two successive injections and is denoted by:

$$
\tau_{\text {cyc }}=\tau_{2}-\tau_{1}
$$

The cut time of component 1 is the time at which fractionation of this component stops. The following equation is used to calculate the cut time $\tau_{c u t}$ of component 1 :

$$
\operatorname{Pur}=\frac{\int_{\tau_{1}}^{\tau_{\mathrm{cut}}} c_{\mathrm{av}, 1}(x=1, \tau) d \tau}{\int_{\tau_{1}}^{\tau_{\mathrm{cut}}}\left[c_{\mathrm{av}, 1}(x=1, \tau)+c_{\mathrm{av}, 2}(x=1, \tau)\right] d \tau},
$$

where $c_{\mathrm{av}}=c_{\mathrm{inj}, 1} C_{\mathrm{av}}$ is defined by Eq. (43). The required peak area based purity was set equal to $99 \%$. A reduced productivity $\operatorname{Pr}$ is the amount of desired compound produced per time cycle. For component 1, it is defined as:

$$
\operatorname{Pr}=\frac{\int_{\tau_{1}}^{\tau_{c u t}} c_{\mathrm{av}, 1}(x=1, \tau) d \tau}{\tau_{c y c}} .
$$

This reduced productivity can be easily back transformed to the usual dimensional form by multiplying with the volumetric flow rate. The recovery yield is the ratio of the amount of desired component in purified fraction and the amount injected at the column inlet. For 
component 1, it is expressed as:

$$
Y=\frac{\int_{\tau_{1}}^{\tau_{\text {cut }}} c_{\mathrm{av}, 1}(x=1, \tau) d \tau}{\int_{\tau_{1}}^{\tau_{2}} c_{\mathrm{av}, 1}(x=1, \tau) d \tau} .
$$

\section{Numerical test problems}

In this section, several test problems of the linear and nonlinear liquid chromatography are considered. In the case of linear isotherm, the semi-analytical solutions and moments derived in Sections 3 and 4 are validated. The high resolution finite volume scheme (HR-FVS) discussed in Section 5 is also applied to numerically approximate the model equations for verifying the correctness of the semi-analytical results. In the case of nonlinear isotherm, the

solutions of the model equations are numerically approximated by the same HR-FVS. In all test problems, we have used $50 \times 20 \times 20$ grid points for the HR-FVS. In the considered test problems, the effects of the core radius fraction $\rho_{\text {core }}$ and $c_{\mathrm{inj}, i}$ are analyzed. Moreover, the effects of $P e_{z}, P e_{\rho}, \zeta$, and $\eta$ characterizing the axial dispersion, radial dispersion, film mass transfer resistance, and intraparticle diffusion resistance, are analyzed on the elution profiles and moments. For the case of nonlinear isotherm, one, two and three components mixture are examined for two different values $(0$ and 0.8$)$ of the core radius fraction $\rho_{\text {core. Unless }}$ otherwise stated, the parameters used in the test problems are given in Table 1. All these parameters are chosen in accordance with ranges typically encountered in HPLC applications [24]. However, we must point out that these parameters do not belong to any particular experiment.

\subsection{Linear isotherm: effects of the core radius fraction $\rho_{\text {core }}$ on the elution profiles}

Figures 2 (a) and (b) shows a comparison of the concentration profiles for different core radius fractions including fully porous beads. As $\rho_{\text {core }}$ increases from 0 (fully porous beads) to 0.85 (beads with a thin shell), the elution profiles becomes sharper, i.e. efficiency increases and this reduces the residence time in the column i.e., capacity decreases. The visible sharpening and the increased symmetry of the peaks in Figure 2 are due to the reduced intraparticle diffusional mass transfer resistance. The shorter retention times are due to the loss of binding sites with a larger $\rho_{\text {core }}$ value, resulting in less interaction between the mobile and the stationary phases for adsorption and desorption. Also, Figure 2 (b) shows that the semi-analytical and numerical solutions of HR-FVS are in good agreement for all values of $\rho_{\text {core }}$. Table 2 shows CPU times 
of both analytical and HR-FVS solutions, as well as the $L^{1}$-errors in time of the HR-FVS at the outlet of the column and at the center of cross-sectional area, i.e. at $x=1$ and $\rho=0$. Here, the following formula was used

$$
L^{1}-\text { error }=\sum_{n=1}^{N_{T}}\left|C_{A}^{n}-C_{N}^{n}\right| \Delta t,
$$

where $C_{A}^{n}:=C_{A}\left(x=1, \rho=0, \tau_{n}\right)$ denotes the analytical solution at the column outlet and at the center of cross-sectional area at time $\tau_{n}$ and $C_{N}^{n}:=C_{N}\left(x=1, \rho=0, \tau_{n}\right)$ represents the corresponding numerical solution. Moreover, $N_{T}$ denotes the total number of time steps and $\Delta t$ represents the time step size. The results indicates the good accuracy of our proposed numerical algorithm and provides further confidence on the derived semi-analytical solutions.

\subsection{Linear isotherm: effects of kinetic parameters}

Figures 3 to 6 show the effects of model parameters $P e_{z}, P e_{\rho}, \zeta$ and $\eta$ on the elution profiles for two different values of $\rho_{\text {core }}$. Figure 3 shows the effects of the radial Peclet number on the elution profiles for core-shell particles $\left(\rho_{\text {core }}=0.8\right)$. The results were obtained for an injection through the inner zone using smaller and larger Peclet numbers (i.e. $P e_{\rho}=1.5$ and $\left.P e_{\rho}=150\right)$ which corresponds to columns of smaller and larger diameters, respectively. The other parameters are exactly the same as given in Table 1. In the case of larger radial Peclet number (i.e. larger diameter column), the difference between 1D (see [22]) and 2D models is more pronounced, compared to the case of smaller radial Peclet number (i.e. smaller diameter column). Also, the 1D plots (i.e. Figure 3 (c)) of the radial concentration profiles at the middle of the column $(\mathrm{x}=0.5)$ are obtained, using two different values of $P e_{\rho}$ and keeping $P e_{z}=$ 600 fixed. It can be observed that the imposed step profile deteriorated faster for the small $P e_{\rho}$ (or larger radial dispersion coefficient $D_{r}$ ) and the limiting case, $P e_{\rho}=1.5$ corresponds to the elimination of injection profiles. These calculations confirm that the model correctly describe the evolution of radial mass transfer and, thus, provides more detailed insight into the column dynamics.

Figures 4 show the same case study for outer zone injection as considered in Figures 3 for inner zone injection. Similar conclusions can obviously be drawn as in the case of inner zone injection. Figures 5 (a) and (b) show the effects of the axial Peclet number for $\rho_{\text {core }}=0$ and $\rho_{\text {core }}=0.8$, respectively. It can be seen that if the axial dispersion is more rate limiting, the peak becomes wider and its height decreases. In other words, the column efficiency reduces 
whenever the value of $P e_{z}$ is decreased. Also, the effects of the different values of $\rho_{\text {core }}$ can clearly be seen on these plots. Figure 6 show that for small values of $\zeta$ and $\eta$, the mass transfer and diffusion rates are relatively slow. Thus, the peak are tailed and broadened.

\subsection{Linear isotherm: discussion on analytical moments and HETP-curves}

Figure 7 gives a quantitative comparison of analytical and numerical averaged moments for different core radius fractions $\rho_{\text {core. }}$ It can be seen that as $\rho_{\text {core }}$ increases from 0 (fully porous beads) to 0.8 (beads with a thin shell), the values of the moments are decreasing. With an increase of $\rho_{\text {core }}$, the first moment $\mu_{1}$, describing the retention time of the elution profile, decreases. As $\rho_{\text {core }}$ increases, the profiles become sharper, giving a reduction in the second central moment $\mu_{2}^{\prime}$ which measures the variance of the elution profiles. The third central moment $\mu_{3}^{\prime}$, which measures the asymmetry of the elution profiles, is also decreasing with increasing $\rho_{\text {core }}$. Furthermore, the fourth central moment $\mu_{4}^{\prime}$, which measures the tailedness or peakedness of the the elution profiles also decreases with increasing $\rho_{\text {core }}$. A good agreement can be seen between analytical and numerical results. The scale of Figure 7 (d) ranges from 0 to 8000 and the large analytical expression of $\mu_{4}^{\prime}$ involves several arithmetic operations. Thus, large values of the $\mu_{4}^{\prime}$ and errors in the calculations of both analytical and numerical solutions lead to this visible difference between the two solutions. However, this difference is acceptable for this high order complicated moment, as these results are obtained after several truncation and round off errors. Figure 8 shows inner zone injection effects of the radial peclet number $P e_{\rho}$ on the local moments for $\rho_{\text {core }}=0.8$. The plots of this figure show that moments approach to constant values along the radial coordinate for smallest value of $P e_{\rho}$ or largest $D_{r}$. For the smallest value of $P e_{\rho}=1.5$, the results correspond to the $1 \mathrm{D}$ results presented in Appendix A of Qamar et al. [20]. Since the concentration is injected via the inner cylindrical core, all moments do not change close to the column center. The changes clearly occur in the outer section. Although, trends of the moments are similar, on inspecting closer the y-axis, the magnitudes reveal that higher moment change more significantly with changing the $P e_{\rho}$. Similar trends were also observed in the case of injection through outer zone. These results are in agreement with the results in Figure 3 (c). For the effects of $P e_{\rho}$ on fully porous particles, see Qamar et al. [41]. Figures 9 (a)-(d) shows plots of the local moment on the radial coordinate this time for $\rho_{\text {core }}=0,0.5$ and 0.8 . It can be seen that the values of the moments are decreasing as the value of $\rho_{\text {core }}$ is increased from 0 to 0.8 . Figure 
9 (e) shows the results of the plotted plate heights HETP (c.f. Eq. (52)) as a function of the velocity $u$. The illustrated calculations were done for the Dirichlet $\mathrm{BC}$ and different values of $\rho_{\text {core }}$. It can be seen that an increase in $\rho_{\text {core }}$, (i.e. a decrease of the thickness of the porous layer), causes smaller HETP-values. For a detailed result and analysis of the HETP curves, see Qamar et al. [22].

\subsection{Nonlinear isotherms: one, two and three components mixture}

Figure 10 show the results for nonlinear isothermal model case $(b=10)$, for $\rho_{\text {core }}=0,0.6$ and 0.85. Comparing these results with the results of Figure 2, it is apparent that the nonlinear effects caused further reduction in the retention times and more pronounced peak tailings. Figure 11 shows the effect of $\rho_{\text {core }}$ on the retention times and band broadening of the elution profiles for a two-component mixture. It can be seen that for fully porous particles the overlap in the elution profiles is very significant and the separation of the two peaks is not achieved in this case. However, for core-shell particles with $\rho_{\text {core }}=0.8$ the elution peaks are sharper, retention times of both components are shorter and the resolution is much better. It can also be observed that separation of the two peaks is almost achieved due to the decrease in band broadening. Moreover, the aggregate dimensionless time required for both elution peaks to be fully observed is significantly reduced from 140 for fully porous particles to 60 for $\rho_{\text {core }}=0.8$, thereby offering larger productivity in a repetitive batch regime. Figure 12 shows the effect of $\rho_{\text {core }}$ on retention times and band broadening of the elution profiles for a three-component mixture. The same situation seen in the two-component case (c.f. Figure 11) is observed here as $\rho_{\text {core }}=0.8$ improved the separation of the mixtures. The aggregate dimensionless time required for the three elution peaks to be fully observed was also significantly reduced from 140 for $\rho_{\text {core }}=0$ to 60 for $\rho_{\text {core }}=0.8$.

\subsection{Process performance assessment}

Figure 13 shows the plot of $\tau_{c u t}, \tau_{c y c}, P r$, and $Y$ (c.f. $\left.(73)(76)\right)$ over $\rho_{\text {core }}$ It can be seen that the cut time decreases from 34.38 for fully porous particles to 13.01 for core-shell particles having $\rho_{\text {core }}=0.875$. Similarly, the cycle time decreases from 134.32 to 37.81 . The productivity increases till $\rho_{\text {core }}=0.8$ and decreases afterwards. Also, the yield Y increases till $\rho_{\text {core }}=0.85$ and a little decreases at $\rho_{\text {core }}=0.875$. Figure 14 shows the effect of the injection concentration $c_{\text {inj }}$ on the parameters $\tau_{\text {cut }}, \tau_{\text {cyc }}, P r$, and $Y$ (c.f. Eqs. (73)-(76)) for 
four different values of $\rho_{\text {core }}$. Here, we have chosen $c_{\mathrm{inj}, 1}=c_{\mathrm{inj}, 2}$. On increasing $c_{\mathrm{inj}, 1}$, the productivity increases and achieves a maximum value after which it maintains a steady value for all values of $\rho_{\text {core }}$. Table 3 lists the maximum values of productivity along with other parameters at the given values of $\rho_{\text {core }}$ and $c_{\text {inj,1 }}$. It can be seen in Table 3 and Figure 14 that maximum values of productivity were achieved in the range of $5.0 \mathrm{~g} / \mathrm{l} \leq c_{\mathrm{inj}, 1} \leq 9.0 \mathrm{~g} / \mathrm{l}$ for $0 \leq \rho_{\text {core }} \leq 0.85$. In this range, the maximum productivity is found at $\rho_{\text {core }}=0.8$ and $c_{\mathrm{inj}, 1}=8.0$. It should be noted that an increase in the feed concentration is equivalent to the increase of $b_{j}$ as shown in the isotherm expression given by Eq. (6). The effects of two kinetic parameters (a) the external mass transfer resistance, expressed as $\zeta$ (Biot number) and (b) the intraparticle diffusion resistance, expressed as $\eta$, are further evaluated. Figure 15 shows that the optimal values of $\rho_{\text {core }}$ depend on the kinetic parameter $\zeta$. Taking the results of Figures 13(c) and (d) generated for $\zeta=50$ as a reference, two other Biot numbers were considered $(\zeta=15$ and $\zeta=150)$. A decrease and an increase of the mass transfer rate around the particles changed in the same way the ratio between external and internal mass transfer resistances. Figure 15(a) shows that the optimal $\rho_{\text {core }}$ values, which lead to the highest productivity for collecting the first eluting component, move to larger values on decreasing $\zeta$, i.e. to thinner shell layers. Simultaneously, the recovery drops with a decrease in mass transfer rate. The differences in productivity and yield for Biot numbers of 50 or 150 are significant as compared to the differences between Biot numbers of 15 and 50. This indicates that for $\zeta=50$ the effect of the transport through the laminar boundary layer is not negligible. Band shapes under such conditions are essentially controlled by internal mass transfer resistances and axial dispersion. Lastly, the results displayed in Figure 15(c) and (d) show the dependence of optimal $\rho_{\text {core }}$ values on the parameter $\eta$. Once again, taking the results of Figures 13(c) and (d) generated for $\eta=2.0$ as a reference, two other $\eta$ numbers were considered (i.e. $\eta=0.5,2.5)$. Figure $15(\mathrm{c})$ shows that the optimal $\rho_{\text {core }}$ values, which lead to the highest productivity for collecting the first eluting component, move to lower values on increasing $\eta$. For $\eta=2.5$ the optimum $\rho_{\text {core }}$ is roughly 0.8 at a higher output than for $\eta=2.0$. Thus, faster transfer rates in the shell allow us to thicken this layer. Simultaneously, the recovery drops monotonously with a decrease in $\eta$ value. It should be emphasized that these results in Figure 15 are just valid for the given feed composition. Changes in the optima would occur for other injections concentrations as illustrated in Figure 14. With the model applied and the accurate 
solution method investigated, the impact of all other relevant parameters could be studied in detail. The selected case studies of this manuscript clearly indicate that a full optimization of core-shell particles for applications in nonlinear preparative chromatography is a challenging task. For application in preparative chromatography, a rational optimization needs to be carefully considered, besides the geometric, kinetic and thermodynamic parameters, also the operating parameters, in particular the injection concentrations.

\section{Conclusion}

Analytical and numerical solutions of a two-dimensional general rate model were derived to simulate single and multi-component solutes transports in the chromatographic columns of cylindrical geometry packed with core-shell particles. For the linear model equations, the analytical solutions were derived by successively applying the finite Hankel and Laplace transformations. The solutions were derived for two sets of boundary conditions and considering injections through inner and outer zones of the column inlet cross section. The developed analytical solutions extend our previous analysis by incorporating the influences of mass transfer coefficient, intraparticle diffusion, and longitudinal and radial dispersion coefficients. The derived analytical solutions were compared with the numerical solutions of a high resolution flux limiting finite volume scheme. Typical case studies were considered and analyzed. Such analytical solutions are useful to perform initial or approximated analysis of the field scale scenarios, to analyze the underlying transport process, to do sensitivity analysis, and to validate numerical solutions, and to determine longitudinal and radial dispersion coefficients from experimental moments. For the nonlinear model equations, the same high resolution finite volume scheme was used to obtain approximate solutions of the nonlinear model for one, two and three components mixture. The current 2D-GRM is more useful than 1D models when the radial dispersion is rate limiting.

\section{Appendix A}

In this work, the first four moments are calculated for both Dirichlet (Eq.(36) and Eq.(44)) and Danckwert boundary conditions (Eq.(41) and Eq.(44)).

Case 1: Rectangular concentration pulse injection as Dirichlet BCs (Eq. (31) and $(32))$ : 
The zeroth moment: It is obtained for $i=0$ :

Let us define

$$
v=\sqrt{P e_{z}^{2}+\frac{4 P e_{z} \lambda_{n}^{2}}{P e_{\rho}}}
$$

Then

$$
\mu_{0, H}=\tau_{\text {inj }} F\left(\lambda_{n}\right) \exp \left(\frac{P e_{z}-v}{2}\right),
$$

where, $F\left(\lambda_{n}\right)$ is given by Eqs. (33) and (34).

First moment: for $i=1$ :

$$
\mu_{1, H}=\left[\frac{\tau_{i n j}}{2}+\frac{P e_{z}\left(1+\left(1-\rho_{\mathrm{core}}\right)^{2} a^{*} F\right)}{v}\right] \mu_{0, H},
$$

where $a^{*}$ is given by Eq. (24).

Second moment: for $i=2$ :

$$
\begin{aligned}
\mu_{2, H}= & {\left[\frac{\tau_{\text {inj }}^{2}}{3}+\frac{P e_{z}\left(1+\left(1-\rho_{\text {core }}\right)^{2} a^{*} F\right)}{v} \tau_{i n j}+\frac{2 P e_{z}^{2}+P e_{z}^{2} v}{v^{3}}\left(1+\left(1-\rho_{\text {core }}\right)^{2} a^{*} F\right)^{2}\right.} \\
& \left.+\frac{2 P e_{z}^{2}\left(1-\rho_{\text {core }}\right)^{4} a^{*^{2}} F(\zeta+5)}{15 v \eta \zeta}\right] \mu_{0, H} .
\end{aligned}
$$

Third moment: for $i=3$ :

$$
\begin{aligned}
\mu_{3, H}= & {\left[\frac{\tau_{\text {inj }}^{3}}{4}+\frac{\tau_{\text {inj }}^{2} P e_{z}}{v}\left(1+\left(1-\rho_{\text {core }}\right)^{2} a^{*} F\right)+\left(\frac{3 P e_{z}^{2}(2+v)}{2 v^{3}}\left(1+\left(1-\rho_{\text {core }}\right)^{2} a^{*} F\right)^{2}\right.\right.} \\
& \left.+\frac{P e_{z}\left(1-\rho_{\text {core }}\right)^{4} a^{*^{2}} F(\zeta+5)}{5 v \eta \zeta}\right) \tau_{i n j}+\frac{12 P e_{z}^{3}+P e_{z}^{3} v^{2}+6 P e_{z}^{3} v}{v^{5}}\left(1+\left(1-\rho_{\text {core }}\right)^{2} a^{*} F\right)^{3} \\
& +\frac{2 P e_{z}^{2}\left(1+\left(1-\rho_{\text {core }}\right)^{2} a^{*} F\right)\left(1-\rho_{\text {core }}\right)^{4} a^{*^{2}} F(\zeta+5)(v+2)}{5 v^{3} \zeta \eta} \\
& \left.+\frac{2 P e_{z}\left(1-\rho_{\text {core }}\right)^{6} a^{*^{3}} F\left(2 \zeta^{3}+14 \zeta+35\right)}{105 v \zeta^{2} \eta^{3}}\right] \mu_{0, H}
\end{aligned}
$$

Fourth moment: for $i=4$

$$
\begin{aligned}
\mu_{4, H}= & {\left[\frac{\tau_{\text {inj }}^{4}}{5}+\frac{P e_{z}\left(1+\left(1-\rho_{\text {core }}\right)^{2} a^{*} F\right)}{v} \tau_{\text {inj }}^{3}+\left(\frac{2 P e_{z}^{2}(2+v)\left(1+\left(1-\rho_{\text {core }}\right)^{2} a^{*} F\right)^{2}}{v^{3}}\right.\right.} \\
& \left.+\frac{4 P e_{z}\left(1-\rho_{\text {core }}\right)^{4} a^{*^{2}} F(\zeta+5)}{15 v \zeta \eta}\right) \tau_{\text {inj }}^{2}+\left(\frac{2\left(12 P e_{z}^{3}+P e_{z}^{3} v^{2}+6 P e_{z}^{3} v\right)}{v^{5}}\left(1+\left(1-\rho_{\text {core }}\right)^{2} a^{*} F\right)^{3}\right.
\end{aligned}
$$




$$
\begin{aligned}
& \left.+\frac{4 P e_{z}^{2}\left(1-\rho_{\text {core }}\right)^{4} a^{*^{2}} F(\zeta+5)}{5 v^{3} \zeta \eta}(2+v)+\frac{4 P e_{z}\left(1-\rho_{\text {core }}\right)^{6} a^{*^{3}} F\left(2 D^{*^{2}}+14 \zeta+35\right)}{105 v \zeta^{2} \eta^{2}}\right) \tau_{\text {inj }} \\
& +\frac{P e_{z}^{4}\left[120+12 v^{2}+\left(v^{2}+60\right) v\right]}{v^{7}}\left(1+\left(1-\rho_{\text {core }}\right)^{2} a^{*} F\right)^{4} \\
& +\frac{4 P e_{z}^{3}\left(1+\left(1-\rho_{\text {core }}\right)^{2} a^{*} F\right)^{2}\left(1-\rho_{\text {core }}\right)^{4} a^{*^{2}} F(\zeta+5)}{5 v^{5} \zeta \eta}\left(v^{2}+6 v+12\right) \\
& +\frac{8 P e_{z}^{2}\left(1+\left(1-\rho_{\text {core }}\right)^{2} a^{*} F\right)\left(1-\rho_{\text {core }}\right)^{6} a^{*^{3}} F\left(2 \zeta^{2}+14 \zeta+35\right)}{105 v^{3} \zeta^{2} \eta^{2}}(v+2) \\
& +\frac{4 P e_{z}^{2}\left(1-\rho_{\text {core }}\right)^{8} a^{*^{4}} F^{2}(\zeta+5)^{2}}{75 v^{3} \zeta^{2} \eta^{2}}(v+2)+\frac{8 P e_{z}\left(1-\rho_{\text {core }}\right)^{8} a^{*^{4}} F}{1575 v \zeta^{3} \eta^{3}} . \\
& \left.\left(17 \zeta^{2}+105 \zeta+175\right)\right] \mu_{0, H} .
\end{aligned}
$$

Case 2: Rectangular concentration pulse injection as Danckwert BC:(Eqs. (37) and (38)).

Zeroth moment: By defining

$$
w=\sqrt{1+\frac{4 \lambda_{n}^{2}}{P e_{z} P e_{\rho}}},
$$

we have

$$
\mu_{0, H}=\frac{4 \tau_{\mathrm{inj}} F\left(\lambda_{n}\right) e^{P e_{z}} w}{(w+1)^{2} e^{\frac{P e_{z}(w+1)}{2}}-(w-1)^{2} e^{-\frac{P e_{z}(w-1)}{2}}} .
$$

\section{First Moment:}

The first moment for $i=1$ is given as

$$
\mu_{1, H}=\left[\frac{\tau_{\text {inj }}}{2}+\frac{\left(1+\left(1-\rho_{\text {core }}\right)^{2} a^{*} F\right)}{P e_{z} w}\left(\frac{\psi_{3} \psi_{1}-\psi_{4} \psi_{2}}{\psi_{7}}-\frac{2}{w}\right)\right] \mu_{0, H},
$$

where

$$
\begin{aligned}
& \psi_{1}=e^{\frac{P e_{z}(w+1)}{2}}, \quad \psi_{2}=e^{\frac{-P e_{z}(w-1)}{2}}, \quad \psi_{3}=4(w+1)+P e_{z}(w+1)^{2}, \\
& \psi_{4}=4(w-1)-P e_{z}(w-1)^{2}, \quad \psi_{5}=w+1, \quad \psi_{6}=w-1, \\
& \psi_{7}=(w+1)^{2} e^{\frac{P e z(w+1)}{2}}-(w-1)^{2} e^{\frac{-P e_{z}(w-1)}{2}} .
\end{aligned}
$$

\section{Second Moment:}

Using the above definitions and equations for $i=2$, we have obtain

$$
\begin{aligned}
\mu_{2, H}= & {\left[\frac{\tau_{\text {inj }}^{2}}{3}+\left[\frac{\left(1+\left(1-\rho_{\text {core }}\right)^{2} a^{*} F\right)}{P e_{z} w}\left(\frac{\psi_{3} \psi_{1}-\psi_{4} \psi_{2}}{\psi_{7}}-\frac{2}{w}\right)\right] \tau_{\text {inj }}+\frac{2\left(1+\left(1-\rho_{\text {core }}\right)^{2} a^{*} F\right)^{2}}{P e_{z}^{2} w^{2}}\left(\frac{\psi_{3} \psi_{1}-\psi_{4} \psi_{2}}{\psi_{7}}\right)^{2}\right.} \\
& -\frac{4\left(1+\left(1-\rho_{\text {core }}\right)^{2} a^{*} F\right)^{2}}{P e_{z}^{2} w^{3}}\left(\frac{\psi_{3} \psi_{1}-\psi_{4} \psi_{2}}{\psi_{7}}\right)+\frac{2\left(1+\left(1-\rho_{\text {core }}\right)^{2} a^{*} F\right)\left(\psi_{5}^{2} \psi_{1}+\psi_{6}^{2} \psi_{2}\right)}{P e_{z} w^{3} \psi_{7}}
\end{aligned}
$$




$$
\begin{aligned}
& +\frac{8\left(1+\left(1-\rho_{\text {core }}\right)^{2} a^{*} F\right)^{2}\left(\psi_{1}-\psi_{2}\right)}{P e_{z}^{2} w^{2} \psi_{7}}+\frac{\left(1+\left(1-\rho_{\text {core }}\right)^{2} a^{*} F\right)^{2}}{w^{2}} \\
& +\frac{2}{15}\left(\frac{\left(\psi_{3} \psi_{1}-\psi_{4} \psi_{2}\right)-\left(\psi_{5}^{2} \psi_{1}+\psi_{6}^{2} \psi_{2}\right)}{P e_{z} w \zeta \eta \psi_{7}}\right)\left(1-\rho_{\text {core }}\right)^{4} a^{* 2} F(\zeta+5) \\
& -\frac{8\left(1+\left(1-\rho_{\text {core }}\right)^{2} a^{*} F\right)^{2}\left(\psi_{6} \psi_{2}+\psi_{5} \psi_{1}\right)}{P e_{z} w^{2} \psi_{7}}+\frac{8\left(1+\left(1-\rho_{\text {core }}\right)^{2} a^{*} F\right)^{2}\left(\psi_{6} \psi_{2}-\psi_{5} \psi_{1}\right)}{P e_{z}^{2} w^{3} \psi_{7}} \\
& +\frac{4}{P e_{z} w^{2}}\left(\frac{\left(1-\rho_{\text {core }}\right)^{4} a^{* 2} F(\zeta+5)}{15 \zeta \eta}-\frac{\left(1+\left(1-\rho_{\text {core }}\right)^{2} a^{*} F\right)^{2}}{P e_{z} w^{2}}\right) \\
& \left.+\frac{8\left(1-\rho_{\text {core }}\right)^{4} a^{* 2} F(\zeta+5)\left(\psi_{5} \psi_{1}-\psi_{6} \psi_{2}\right)}{15 P e_{z} w \zeta \eta \psi_{7}}\right] \mu_{0, H} .
\end{aligned}
$$

\section{Third Moment:}

Let us define

$$
\begin{aligned}
\psi_{8} & =\frac{\psi_{3} \psi_{1}-\psi_{4} \psi_{2}}{P e_{z} w \psi_{7}}\left(1+\left(1-\rho_{\text {core }}\right)^{2} a^{*} F\right), \\
\psi_{9} & =\frac{\left(1-\rho_{\text {core }}\right)^{4} a^{* 2} F(\zeta+5)}{5 P e_{z} w^{2} \zeta \eta}-\frac{3\left(1+\left(1-\rho_{\text {core }}\right)^{2} a^{*} F\right)^{2}}{P e_{z}^{2} w^{4}}, \quad \psi_{10}=\frac{\psi_{5}^{2} \psi_{1}+\psi_{6}^{2} \psi_{2}}{P e_{z} w^{3} \psi_{7}}, \\
\psi_{11} & =\frac{\psi_{6} \psi_{2}+\psi_{5} \psi_{1}}{P e_{z} w^{2} \psi_{7}}, \quad \psi_{12}=\frac{\psi_{1}-\psi_{2}}{P e_{z}^{2} w^{2} \psi_{7}}, \quad \psi_{13}=\frac{\psi_{6} \psi_{2}-\psi_{5} \psi_{1}}{P e_{z} w^{2} \psi_{7}}, \\
\psi_{14} & =\left[\frac{\left(\psi_{3} \psi_{1}-\psi_{4} \psi_{2}\right)-\left(\psi_{5}^{2} \psi_{1}-\psi_{6}^{2} \psi_{2}\right)}{5 P e_{z} w \zeta \eta \psi_{7}}\right]\left(1-\rho_{\text {core }}\right)^{4} a^{* 2} F, \\
\psi_{15} & =\frac{\left(1-\rho_{\text {core }}\right)^{4} a^{* 2} F\left(\psi_{5} \psi_{1}-\psi_{6} \psi_{2}\right)}{5 P e_{z} w \zeta \eta \psi_{7}}, \quad \psi_{16}=\frac{\left(1+\left(1-\rho_{\text {core }}\right)^{2} a * F\right)\left(\psi_{1}+\psi_{2}\right)}{P e_{z}^{2} w^{2} \psi_{7}} .
\end{aligned}
$$

Then we have

$$
\begin{aligned}
\mu_{3, H}= & {\left[\frac{\tau_{\text {inj }}^{3}}{4}-\left(\psi_{8}-\frac{2}{P e_{z} w^{2}}\right) \tau_{\text {inj }}^{2}-\left[\frac{6\left(1+\left(1-\rho_{\text {core }}\right)^{2} a^{*} F\right)}{P e_{z} w} \psi_{8}+3\left(1+\left(1-\rho_{\text {core }}\right)^{2} a^{*} F\right) \psi_{10}+(\zeta+5) \psi_{14}\right.\right.} \\
& \left.+4(\zeta+5) \psi_{15}+4 \psi_{9}+3 \psi_{8}^{2}-\left(1+\left(1-\rho_{\text {core }}\right)^{2} a^{*} F\right)^{2}\left(12 \psi_{11}-12 \psi_{12}-\frac{3}{2 w^{2}}-\frac{12 \psi_{13}}{P e_{z}}\right)\right] \tau_{\text {inj }} \\
& +\left(6 \psi_{8}-\frac{6\left(1+\left(1-\rho_{\text {core }}\right)^{2} a^{*} F\right)}{P e_{z} w^{2}}\right)\left[2\left(1+\left(1-\rho_{\text {core }}\right)^{2} a^{*} F\right) \psi_{10}-\left(1+\left(1-\rho_{\text {core }}\right)^{2} a^{*} F\right)^{2}\right. \\
& \left.\left(8 \psi_{11}-8 \psi_{12}-\frac{1}{w^{2}}-\frac{8 \psi_{13}}{P e_{z} w}\right)-\frac{2\left(1-\rho_{\text {core }}\right)^{4} a^{* 2} F P e_{z} w^{2}(\zeta+5) \psi_{10}}{15 \zeta \eta}+\frac{8}{3}(\zeta+5) \psi_{15}\right] \\
& +\left(1+\left(1-\rho_{\text {core }}\right)^{2} a^{*} F\right)\left(\frac{16(\zeta+5) \psi_{15}}{P e_{z} w^{2}}-\frac{4\left(1-\rho_{\text {core }}\right)^{4} a^{* 2} F \psi_{10}}{5 \zeta \eta}-\frac{8\left(1-\rho_{\text {core }}\right)^{4} a^{* 2} F(\zeta+5)}{P e_{z}^{2} w^{4}}\right. \\
& \left.+\frac{24 \psi_{16}}{w}+\frac{2\left(1-\rho_{\text {core }}\right)^{4} a^{* 2} F(\zeta+5) P e_{z} \psi_{13}}{5 \zeta \eta}+\frac{16\left(1-\rho_{\text {core }}\right)^{4} a^{* 2} F(\zeta+5) P e_{z} \psi_{11}}{5 \zeta \eta}+\frac{12 \psi_{8}^{2}}{P e_{z} w^{2}}\right) \\
& +\left(1+\left(1-\rho_{\text {core }}\right)^{2} a^{*} F\right)^{3}\left(12 \psi_{13}+P e_{z} \psi_{10}+\frac{12 \psi_{10}}{P e_{z} w^{2}}+\frac{48 \psi_{13}}{P e_{z}^{2} w^{3}}+\frac{6}{P e_{z} w^{4}}-\frac{48 \psi_{11}}{P e_{z} w^{2}}+\frac{48 \psi_{12}}{P e_{z} w^{2}}\right. \\
& \left.+\frac{24}{P e_{z}^{3} w^{6}}\right)+\left(\frac{35}{2}+7 \zeta+\zeta^{2}\right)\left(\frac{16\left(1-\rho_{\text {core }}\right)^{2} a^{*} F \psi_{15}}{21 \zeta \eta}+\frac{4\left(1-\rho_{\text {core }}\right)^{6} a^{* 3} F \psi_{10} w^{2}}{105 \zeta^{2} \eta^{2}}+\frac{8\left(1-\rho_{\text {core }}\right)^{6} a^{* 3} F}{105 P e_{z} w^{2} \zeta^{2} \eta^{2}}\right) \\
& -\frac{2(\zeta+5)}{5}\left(\frac{\left.8\left(1-\rho_{\text {core })^{4} a^{* 2} F \psi_{12}}^{\zeta \eta}+\frac{\left(1-\rho_{\text {core }}\right)^{4} a^{* 2} F P e_{z} w \psi_{11}}{3 \zeta \eta}\right)-6 \psi_{8}^{3}+4 \psi_{9} \psi_{8}\right] \mu_{0, H} .}{(\mathrm{A}-19)}\right.
\end{aligned}
$$




\section{Fourth Moment:}

First, we also define the following

$$
\begin{aligned}
\psi_{17}= & 2\left(1+\left(1-\rho_{\text {core }}\right)^{2} a^{*} F\right) \psi_{10}-\left(1+\left(1-\rho_{\text {core }}\right)^{2} a^{*} F\right)^{2}\left(8 \psi_{11}-8 \psi_{12}-\frac{1}{w^{2}}-\frac{8 \psi_{13}}{P e_{z} w}\right) \\
& -\frac{2\left(1-\rho_{\text {core }}\right)^{4} a^{* 2} F P e_{z} w^{2}(\zeta+5) \psi_{10}}{15 \zeta \eta}+\frac{8}{3}(\zeta+5) \psi_{15}, \\
\psi_{18}= & \left(1+\left(1-\rho_{\text {core }}\right)^{2} a^{*} F\right)^{3}\left(12 \psi_{13}+P e_{z} \psi_{10}+\frac{12 \psi_{10}}{P e_{z} w^{2}}+\frac{48 \psi_{13}}{P e_{z}^{2} w^{3}}+\frac{6}{P e_{z} w^{4}}-\frac{48 \psi_{11}}{P e_{z} w^{2}}+\frac{48 \psi_{12}}{P e_{z} w^{2}}\right), \\
\psi_{19}= & \left(1+\left(1-\rho_{\text {core }}\right)^{2} a^{*} F\right)\left(\frac{16(\zeta+5) \psi_{15}}{P e_{z} w^{2}}-\frac{4\left(1-\rho_{\text {core }}\right)^{4} a^{* 2} F \psi_{10}}{5 \zeta \eta}+\frac{24 \psi_{16}}{w}+\frac{2\left(1-\rho_{\text {core }}\right)^{4} a^{* 2} F(\zeta+5) P e_{z} \psi_{13}}{5 \zeta \eta}\right. \\
& \left.+\frac{16\left(1-\rho_{\text {core }}\right)^{4} a^{* 2} F(\zeta+5) P e_{z} \psi_{11}}{5 \zeta \eta}\right), \\
\psi_{20}= & \left(\frac{35}{2}+7 \zeta+\zeta^{2}\right)\left(\frac{16\left(1-\rho_{\text {core }}\right)^{2} a^{*} F \psi_{15}}{21 \zeta \eta}+\frac{4\left(1-\rho_{\text {core }}\right)^{6} a^{* 3} F \psi_{10} w^{2}}{105 \zeta^{2} \eta^{2}}\right)+\frac{16\left(1-\rho_{\text {core }}\right)^{4} a^{* 2} F(\zeta+5) \psi_{12}}{5 \zeta \eta}, \\
& \left(1+\left(1-\rho_{\text {core }}\right)^{2} a^{*} F\right)(\zeta+5) a^{* 2} F \\
5 P e_{z}^{2} w^{4} \zeta \eta & \frac{3\left(1+\left(1-\rho_{\text {core }}\right)^{2} a^{*} F\right)^{3}}{P e_{z}^{3} w^{6}}+\frac{8\left(1-\rho_{\text {core }}\right)^{6} a^{* 3} F\left(\frac{35}{2}+7 \zeta+\zeta^{2}\right)}{315 P e_{z} w^{2} \zeta \eta},
\end{aligned}
$$

$$
\begin{aligned}
\psi_{22}= & \frac{15\left(1+\left(1-\rho_{\text {core }}\right)^{2} a^{*} F\right)^{4}}{P e_{z}^{4} w^{8}}-\frac{2\left(1-\rho_{\text {core }}\right)^{4} a^{* 2} F\left(1+\left(1-\rho_{\text {core }}\right)^{2} a^{*} F\right)^{2}(\zeta+5)}{5 P e_{z}^{3} w^{6} \zeta \eta}+\frac{\left(1-\rho_{\text {core }}\right)^{8} a^{* 4} F^{2}(\zeta+5)^{2}}{675 P e_{z}^{2} w^{4} \zeta^{2} \eta^{2}} \\
& -\frac{4\left(1-\rho_{\text {core }}\right)^{6} a^{* 3} F\left(1+\left(1-\rho_{\text {core }}\right)^{2} a^{*} F\right)\left(\frac{35}{2}+7 \zeta+\zeta^{2}\right)}{315 P e_{z}^{2} w^{4} \zeta^{2} \eta^{2}}+\frac{16\left(1-\rho_{\text {core }}\right)^{8} a^{* 4} F^{2}\left(175+105 \zeta+27 \zeta^{2}\right)}{4725 P e_{z} w^{2} \zeta^{3} \eta^{3}},
\end{aligned}
$$

$\psi_{23}=\frac{\psi_{6}^{2} \psi_{2}-\psi_{1} \psi_{5}^{2}}{P e_{z} w^{2} \psi_{7}}$.

Then the fourth moment is expressed as

$$
\begin{aligned}
\mu_{4, H}= & {\left[\frac{\tau_{\text {inj }}^{4}}{5}+\left(\psi_{8}+\frac{2}{P e_{z} w^{2}}\right) \tau_{\text {inj }}^{3}+\left(2 \psi_{17}-\frac{8\left(1+\left(1-\rho_{\text {core }}\right)^{2} a^{*} F\right)^{2} \psi_{13}}{P e_{z} w^{2}}+4 \psi_{8}^{2}-8 \psi_{9}\right) \tau_{\text {inj }}^{2}\right.} \\
& +\left(12 \psi_{17}\left(\frac{1+\left(1-\rho_{\text {core }}\right)^{2} a^{*} F}{P e_{z} w^{2}}-\psi_{8}\right)+16 \psi_{21}+12 \psi_{8}^{3}-8 \psi_{9} \psi_{8}+2\left(\psi_{18}+\psi_{19}+\psi_{20}\right)\right. \\
& \left.-\frac{24\left(1+\left(1-\rho_{\text {core }}\right)^{2} a^{*} F\right) \psi_{8}^{2}}{P e_{z} w^{2}}\right) \tau_{\text {inj }}+8 \psi_{9} \psi_{17}+32 \psi_{8} \psi_{21}+6 \psi_{17}^{2}+16 \psi_{9} \psi_{8}^{2}+24 \psi_{8}^{4}-\frac{16 \psi_{22}}{P e_{z} w} \\
& +\frac{8\left(1+\left(1-\rho_{\text {core }}\right)^{2} a^{*} F\right)}{P e_{z} w^{2}}\left(6 \psi_{8} \psi_{17}-6 \psi_{8}^{3}-\psi_{18}\right)+8 \psi_{8} \psi_{18}-36 \psi_{8}^{2} \psi_{17}+4\left(1+\left(1-\rho_{\text {core }}\right)^{2} a^{*} F\right)^{2}(\zeta+5) \\
& \left(\frac{12\left(1-\rho_{\text {core }}\right)^{4} a^{* 2} F}{5 P e_{z} w^{2} \zeta \eta}\left(\psi_{10}-4 \psi_{11}-4 \psi_{12}\right)+\frac{12 \psi_{15}}{w^{2}}+\frac{P e_{z} \psi_{10}}{5 \psi \eta}+\frac{6\left(1-\rho_{\text {core }}\right)^{4} a^{* 2} F}{5 w \zeta \eta}\left(\psi_{21}+4 \psi_{12}\right)+\frac{4 \psi_{15}}{P e_{z}^{2} w^{4}}\right) \\
& +4\left(1+\left(1-\rho_{\text {core }}\right)^{2} a^{*} F\right)^{4}\left(\frac{36}{P e_{z} w^{3}}\left(\psi_{13}-\psi_{16}\right)+\frac{P e_{z} \psi_{10}}{4 w}+\frac{4 \psi_{10}}{w}+\frac{3}{w^{2}}\left(4 \psi_{12}-\psi_{10}\right)-\frac{30 \psi_{10}}{P e_{z}^{2} w^{4}}\right. \\
& \left.+\frac{120}{P e_{z}^{2} w^{4}}\left(\psi_{11}-\psi_{10}\right)+\frac{120 \psi_{13}}{P e_{z}^{3} w^{5}}+\frac{15 \psi_{21}}{w^{4}}\right)+4\left(1+\left(1-\rho_{\text {core }}\right)^{2} a^{*} F\right)\left(\frac{35}{2}+7 \zeta+\zeta^{2}\right) \\
& \left(\frac{4\left(1-\rho_{\text {core }}\right)^{6} a^{* 3} F}{105 \zeta^{2} \eta^{2}}\left(P e_{z} \psi_{21}+2 \psi_{10}-8 \psi_{11}-8 \psi_{12}\right)+\frac{32\left(1-\rho_{\text {core }}\right)^{2} a^{*} F \psi_{15}}{21 P e_{z} w^{2} \zeta^{2} \eta^{2}}\right)+\frac{4\left(1-\rho_{\text {core }}\right)^{8} a^{* 4} F^{2}(\zeta+5)}{75 \zeta^{2} \eta^{2}}
\end{aligned}
$$




$$
\begin{aligned}
& \left(2(\zeta+5)\left(4 \psi_{11}-\psi_{10}\right)+8 \psi_{12}+P e_{z} \psi_{21}+\frac{8(\psi+5) \psi_{13}}{P e_{z} w}\right) \\
& \left.+\frac{8\left(175+105 \zeta+27 \zeta^{2}\right)}{\zeta^{2} \eta^{2}}\left(\frac{\left(1-\rho_{\text {core }}\right)^{8} a^{* 4} F^{2} P e_{z} w^{2} \psi_{10}}{1575 \zeta \eta}+\frac{4 \psi_{15}}{315\left(1-\rho_{\text {core }}\right)^{2} a^{*} F}\right)\right] \mu_{0, H} .
\end{aligned}
$$

The analytical expressions of solutions and moments have been implemented in Matlab software. The corresponding author of this article is willing to provide these Matlab codes to readers. The soft copies of these Matlab codes will also be available in the library of Max Planck Institute Magdeburg, Germany.

\section{References}

[1] Kirkland, J.J., Truszkowski, F.A., Dilks, C.H., Engel, G.S., 2000. Superficially porous silica microspheres for fast high-performance liquid chromatography of macromolecules. J. Chromatogr. A 890, 3-13.

[2] Fekete, S., Ganzler, K., Fekete, J., 2010. Facts and myths about columns packed with sub-3 $\mu \mathrm{m}$ and sub-2 $\mu \mathrm{m}$ particles. Journal of Pharmaceutical and Biomedical Analysis 51,5664 .

[3] Rissler, R., 2000. Separation of polyesters by gradient reversed-phase high- performance liquid chromatography on a $1.5 \mu$ m non-porous column. J. Chromatogr. A 871, 243258.

[4] Xiang,Y.Q., Yan,B.W., McNeff,C.V., Carr,P.W., Lee,M.L., 2003. Synthesis of micron diameter polybutadiene-encapsulated non-porous zirconia particles for ultrahigh pressure liquid chromatography. J. Chromatogr. A 1002, 7178.

[5] Miyabe, K., 2008. Evaluation of chromatographic performance of various packing materials having different structural characteristics as stationary phase for fast high performance liquid chromatography by new moment equations. Journal of Chromatography A 1183,4964

[6] Manchon, N., Arrigo, M.D., Garcia-Lafuente, A., Guillamon, E., Villares, A., Ramos, A., Martinez, J.A., Rostagno, M.A., 2010. Fast analysis of isoflavones by high-performance liquid chromatography using a column packed with fused-core particles. Talanta 82, 19861994 . 
[7] Wang, C., Soice,N.P.., Ramaswamy,S., Gagnon, B.A., Umana, J., Cotoni, K.A., Bian, N., Cheng, K.-S.C., 2007. Cored anion-exchange chromatography media for anti- body flow-through purification. J. Chromatogr. A 1155, 7484.

[8] Tingyue Gu, Mingqing Liu, Kwok-Shun C. Cheng, Senthilkumar Ramaswamy, Chen Wang, 2011. A general rate model approach for the optimization of the core radius fraction for multicomponent isocratic elution in preparative nonlinear liquid chromatography using cored beads. Chem. Eng. Sci. 66, 3531-3539.

[9] Zhou, X., Shi, Q-H, Bai, S., Sun, Y., 2004. Dense pellicular agarose-glass beads for expanded bed application: fabrication and characterization for effective protein adsorption. Biochemical Engineering Journal 18, 8188.

[10] Ning, J., Kong, F., Li, D., Du, Y., 1998. Preparation of monodisperse agglomerated pellicular anion-exchange resins compatible with high-performance liquid chromatography solvents for ion chromatography. Journal of Chromatography A 793, 193197.

[11] Schuster, S.A., Wagner, B.M., Boyes, B.E., Kirkland, J.J., 2010. Wider pore superficially porous particles for peptide separations by HPLC. Journal of Chromatographic Science 48,566571

[12] Kiss, I., Bacskay, I., Kilr, F., Felinger, A., 2010. Comparison of the mass transfer in totally porous and superficially porous stationary phases in liquid chromatography. Analytical and Bioanalytical Chemistry 397, 13071314.

[13] Zhou, X., Sun, Y., Liu, Z., 2007. Superporous pellicular agaroseglass composite particle for protein adsorption. Biochemical Engineering Journal 34, 99106.

[14] Kaczmarski, K., Guiochon, G., 2007. Modeling of the mass-transfer kinetics in chromatographic columns packed with shell and pellicular particles. Analytical Chemistry 79,46484656 .

[15] Li, P., Yu, J., Xiu, G., Rodrigues, A.E., 2010. A strategy for tailored design of efficient and low-pressure drop packed column chromatography. AIChE. Journal 56, 30913098.

[16] Yang, G., Hu, Z., 1996. Universal theoretical moment expressions for elution and frontal 
chromatography of pellicular ion exchange resins. Reactive and Functional Polymers 31, 2529.

[17] Jian Luo, Weiqing Zhou, Zhiguo Su, Guanghui Ma, Tingyue Gu, 2013. Comparison of fully-porous beads and cored beads in size exclusion chromatography for protein purification. Chem. Eng. Sci. 107, 99-105.

[18] Qamar, S., Bibi, S., Khan, F.U., Shah, M., Javeed, S., Seidel-Morgenstern, A, 2014. Irreversible and Reversible Reactions in a Liquid Chromatographic Column: Analytical Solutions and Moment Analysis. Ind. eng. Chem. Res. 53, 2461.

[19] Bibi, S., Qamar, S., Seidel-Morgenstern, A., 2015. Irreversible and reversible reactive chromatography: Analytical solutions and moment analysis for rectangular pulse injections. J. Chromatogr. A. 1385, 49-62.

[20] Javeed, S., Qamar, S., Ashraf, W., Seidel-Morgenstern, A., Warnecke, G., 2013. Analysis and numerical investigation of two dynamic models for liquid chromatography. Chem. Eng. Sci. 90, 17-31.

[21] Qamar, S., Abbasi, J.N., Javeed, S., Shah, M., Khan, F.U., Seidel-Morgenstern, A., 2013. Analytical solutions and moment analysis of chromatographic models for rectangular pulse injections, J. Chromatogr. A 1315, 92-106.

[22] Qamar, S., Abbasi, J., Mehwish, A., Seidel-Morgenstern, A., 2015. Linear general rate model of chromatography for coreshell particles: Analytical solutions and moment analysis. Chem. Eng. Sci. 137, 352-363.

[23] Leweke, S., von Lieres, E., 2016. Fast arbitrary order moments and arbitrary precision solution of the general rate model of column liquid chromatography with linear isotherm. J. Comput. Chem. Eng. 84, 350-362.

[24] Guiochon, G., Felinger, A., Shirazi, D.G., Katti, A.M., 2006. Fundamentals of preparative and nonlinear chromatography, 2nd ed. ELsevier Academic press, New York.

[25] Kubin, M., 1965. Beitrag zur Theorie der Chromatographie. Collect. Czech. Chem. Commun. 30, 1104-1118. 
[26] Kubin, M., 1965. Beitrag zur Theorie der Chromatographie. 11. Einfluss der Diffusion Ausserhalb und der Adsorption Innerhalb des Sorbens-Korns. Collect. Czech. Chem. Commun. 30, 2900-2907.

[27] Kucera, E., 1965. Contribution to the theory of chromatography: Linear non-equilibrium elution chromatography. J. Chromatogr. A 19, 237-248.

[28] Miyabe, K., Guiochon, G., 2000. Influence of the modification conditions of alkyl bonded ligands on the characteristics of reversed-phase liquid chromatography. J. Chromatogr. A $903,1-12$.

[29] Miyabe, K., Guiochon, G., 2003. Measurement of the parameters of the mass transfer kinetics in high performance liquid chromatography. J. Sep. Sci. 26, 155-173.

[30] Miyabe, K., 2007. Surface diffusion in reversed-phase liquid chromatography using silica gel stationary phases of different C1 and C18 ligand densities. J. Chromatogr. A 1167, 161-170.

[31] Miyabe, K., 2009. Moment analysis of chromatographic behavior in reversed-phase liquid chromatography. J. Sep. Sci. 32, 757-770.

[32] Ruthven, D.M., 1984. Principles of adsorption and adsorption processes, WileyInterscience, New York.

[33] Schneider, P., Smith, J.M., 1968. Adsorption rate constants from chromatography. AIChE J. 14, 762-771.

[34] Suzuki, M., Smith, J.M., 1971. Kinetic studies by chromatography. Chem. Eng. Sci. 26, 221-235.

[35] Suzuki, M., 1973. Notes on determining the moments of the impulse response of the basic transformed equations. J. Chem. Eng. Japan 6, 540-543.

[36] Wolff, H.-J., Radeke, K.-H, Gelbin, D., 1980. Heat and mass transfer in packed beds-IV use of weighted moments to determine axial dispersion coefficient. Chem. Eng. Sci. 34, 101-107. 
[37] Wolff, H.-J., Radeke, K.-H, Gelbin, D., 1980. Weighted moments and the pore-diffusion model. Chem. Eng. Sci. 35, 1481-1485.

[38] Qamar, S., Khan, F.U., Mehmood, Y., Seidel-Morgenstern, A., 2014. Analytical solution of a two-dimensional model of liquid chromatography including moment analysis, Chem. Eng. Sci. 116, 576-589.

[39] S. Parveen, S. Qamar and A. Seidel-Morgenstern, 2015. Two-dimensional nonequilibrium model of liquid chromatography: Analytical solutions and moment analysis, Chem. Eng. Sci., 122, 64-77.

[40] Parveen, S., Qamar, S., Seidel-Morgenstern, 2016. A. Analysis of two-Dimensional nonEquilibrium model of linear reactive Ccromatography considering irreversible and reversible reactions. Ind. Eng. Chem. Res. 55, 2471-2482.

[41] Qamar, S., Uche, D.U., Khan,F.U., Seidel-Morgenstern, A., 2017. Analysis of linear twodimensional general rate model for chromatographic columns of cylindrical geometry. J. Chromatogr. A 1496, 92104.

[42] Qamar, S., Fouzia, A.S., Abasi, J.N. Seidel-Morgenstern, A., 2016. Numerical simulation of nonlinear chromatography with coreshell particles applying the general rate model. Chem. Eng. Sci. 147, 54-64.

[43] Javeed, S., Qamar, S., Seidel-Morgenstern, A., Warnecke, G., 2011. Efficient and accurate numerical simulation of nonlinear chromatographic processes. J. Comput. Chem. Eng. $35,2294-2305$.

[44] Lieres, E. V.; Andersson, J. (2010). A fast and accurate solver for the general rate model of column liquid chromatography. Journal of Computers and Chemical Engineering, 34, 11801191.

[45] Webley, P. A.; He, J. (2000). Fast solution-adaptive finite volume method for PSA/VSA cycle simulation; 1 single step simulation. Journal of Computers and Chemical Engineering, 23, 17011712 .

[46] LeVeque, R.J., 1992. Numerical Methods for Conservation Laws. Birkhaüser Verlag, Bassel, Germany. 
[47] Guiochon, G., 2002. Preparative liquid chromatography. J. Chromatogr. A, 965, 129-161.

[48] Carslaw, H.S., Jaeger, J.C., 1953. Operational methods in applied mathematics, Oxford University Press, Oxford.

[49] Chen, J.-S., Liu, Y.-H., Liang, C.-P., Liu, C.-W., Lin, C.-W., 2011. Exact analytical solutions for two-dimensional advectiondispersion equation in cylindrical coordinates subject to third-type inlet boundary conditions. Adv. Water Resour. 34, 365-374.

[50] Crank, J., 1975. The mathematics of diffusion, 2nd ed. Clarendon Press, Oxford.

[51] Sneddon, I.H., 1972. The use of integral transforms, McGraw-Hill, New York.

[52] Durbin, F., 1974. Numerical Inversion of Laplace Transforms: An efficient improvement to Dubner and Abate's Method. The Computer Journal 17, 371-376.

[53] Rice, R.G., Do, D.D., 1995. Applied mathematics and modeling for chemical engineers. Wiley-Interscience, New York.

[54] Koren, B., 1993. A robust upwind discretization method for advection, diffusion and source terms. In C. B. Vreugdenhil, B. Koren, editors, Numerical Methods for AdvectionDiffusion Problems, Volume 45 of Notes on Numerical Fluid Mechanics, chapter 5, pages 117-138, Vieweg Verlag, Braunschweig.

[55] Cockburn, B., Shu, C. -W. TVB Runge-Kutta local projection discontinuous Galerkin finite element method for conservation laws II, Gerneral framework, Mathematics of Computation, 52 411-435 (1989).

[56] Horváth, K., Felinger, A., 2015. Influence of particle size and shell thickness of coreshell packing materials on optimum experimental conditions in preparative chromatography. J. Chromatogr. A 1407, 100105. 
Table 1: Standard Parameters Used for the Computer Simulation.

\begin{tabular}{|c|c|c|c|c|c|c|c|c|c|c|c|}
\hline Fig. nr. & Comp. nr. & $\epsilon_{p, i}$ & $\epsilon_{b}$ & $P e_{z, i}$ & $P e_{\rho, i}$ & $a_{i}$ & $\eta_{i}$ & $\zeta_{i}$ & $b_{i}$ & $C_{i}$ & $\xi$ \\
\hline $2-5$ & 1 & 0.333 & 0.4 & 600 & 15 & 4 & 2 & 50 & 0 & 1.0 & 450 \\
\hline 6 & 1 & 0.333 & 0.4 & 600 & 15 & 4 & $0.2,2,20$ & $0.5,5,50$ & 0 & 1.0 & 450 \\
\hline $7-9$ & 1 & 0.333 & 0.4 & 600 & 15 & 4 & 2 & 50 & 0 & 1.0 & 450 \\
\hline 10 & 1 & 0.333 & 0.4 & 1500 & 37.5 & 10 & 2 & 50 & 10 & 1.0 & 450 \\
\hline 11 & 1 & & & & & 10 & & & 1.0 & 0.1 & \\
\hline \multirow{3}{*}{12} & 2 & 0.5 & 0.4 & 1500 & 37.5 & 30 & 2 & 50 & 1.0 & 0.1 & 450 \\
\hline & 1 & & & & & 10 & & & 0.5 & 0.1 & \\
\hline & 2 & & & & & 30 & & & 1.5 & 0.1 & \\
\hline \multirow{3}{*}{$13-15$} & 3 & 0.5 & 0.4 & 1500 & 37.5 & 70 & 2 & 50 & 3.5 & 0.4 & 450 \\
\hline & 1 & & & & & 10 & & & 1.0 & 1.0 & \\
\hline & 2 & 0.5 & 0.4 & 1500 & 37.5 & 30 & 2 & 50 & 1.0 & 1.0 & 450 \\
\hline
\end{tabular}

Table 2: Single component elution: CPU times and $L^{1}$-errors in the solutions of Figure 2

\begin{tabular}{|c|c|c|c|}
\hline$\rho_{\text {core }}$ & Analytical CPU(min) & Numerical CPU(min) & $L^{1}$-error $($ HR-FVS $)$ \\
\hline 0 & 3.5 & 11 & $4.95 \times 10^{-5}$ \\
0.6 & 3.6 & 12 & $4.95 \times 10^{-5}$ \\
0.85 & 3.7 & 16 & $5.03 \times 10^{-5}$ \\
\hline
\end{tabular}

Table 3: Optimum values of parameters used for assessment.

\begin{tabular}{|c|c|c|c|c|c|}
\hline$\rho_{\text {core }}$ & $c_{i n j, 1}$ & $\operatorname{Pr}$ & $\mathrm{Y}$ & $\tau_{\text {cyc }}$ & $\tau_{\text {cut }}$ \\
\hline 0 & 9.0 & 0.0027 & 0.85 & 128.82 & 30.14 \\
0.4 & 8.0 & 0.0016 & 0.87 & 113.64 & 29.04 \\
0.8 & 8.0 & 0.0038 & 0.98 & 53.64 & 17.08 \\
0.85 & 5.0 & 0.0023 & 0.99 & 41.88 & 14.08 \\
\hline
\end{tabular}



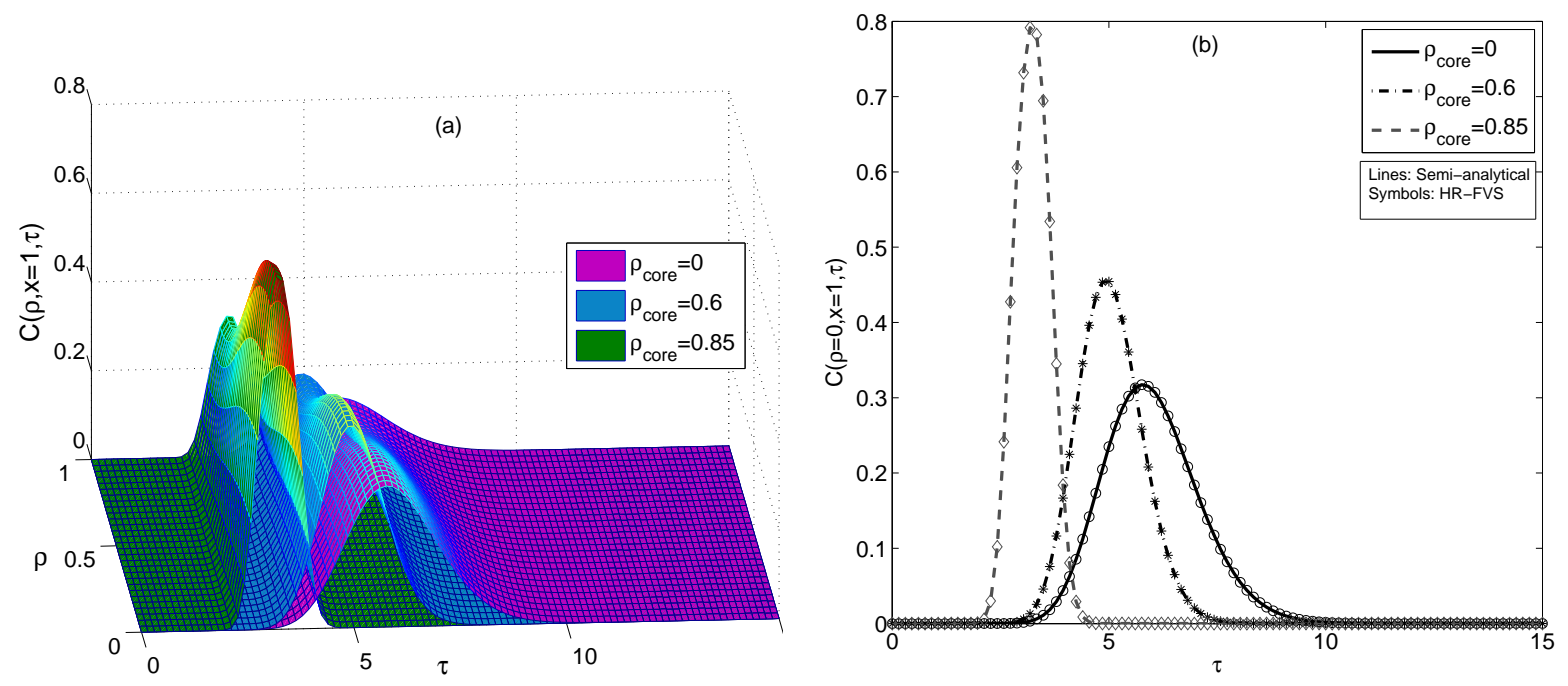

Figure 2: Linear isotherm and inner zone injection: Effect of core-radius fraction on the elution profiles for the Danckwerts BCs. 
Inner zone inj. $\mathrm{Pe}_{\rho}=1.5, \rho_{\text {core }}=0.8$

(a)

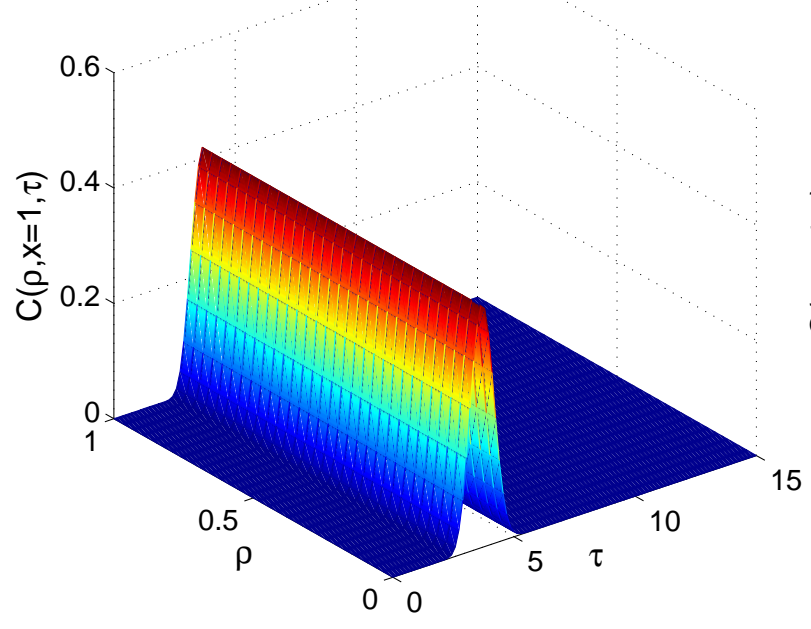

Inner zone inj. $P e_{\rho}=150, \rho_{\text {core }}=0.8$

(b)

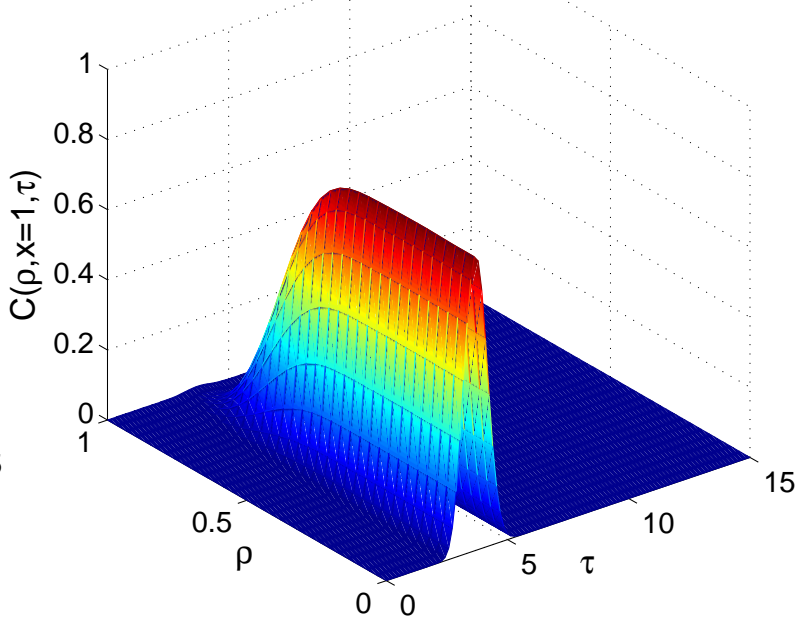

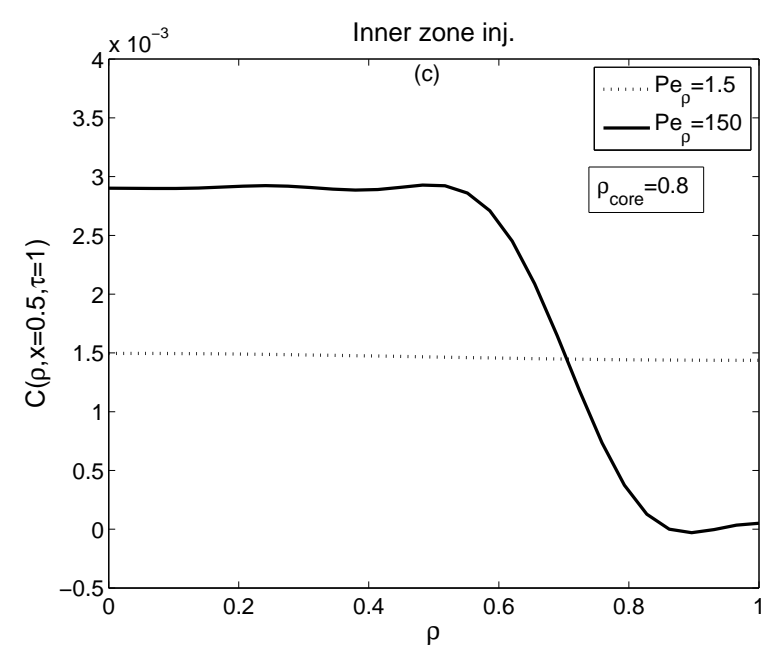

Figure 3: Linear isotherm and inner zone injection: Effects of radial Peclet number $\left(P e_{\rho}\right)$ on the concentration profiles for $\rho_{\text {core }}=0.8$. The $1 \mathrm{D}$ plots in (c) are given at the centre of the column $x=0.5$. The results were obtained using the Danckwerts BC. 
Outer zone inj. $P e_{\rho}=1.5, \rho_{\text {core }}=0.8$

(a)

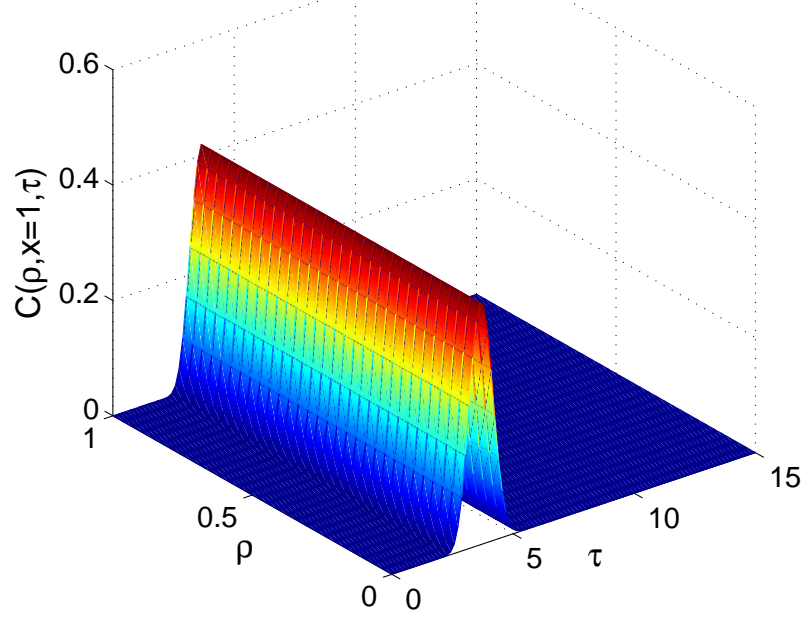

Outer zone inj. $P e_{\rho}=150, \rho_{\text {core }}=0.8$

(b)

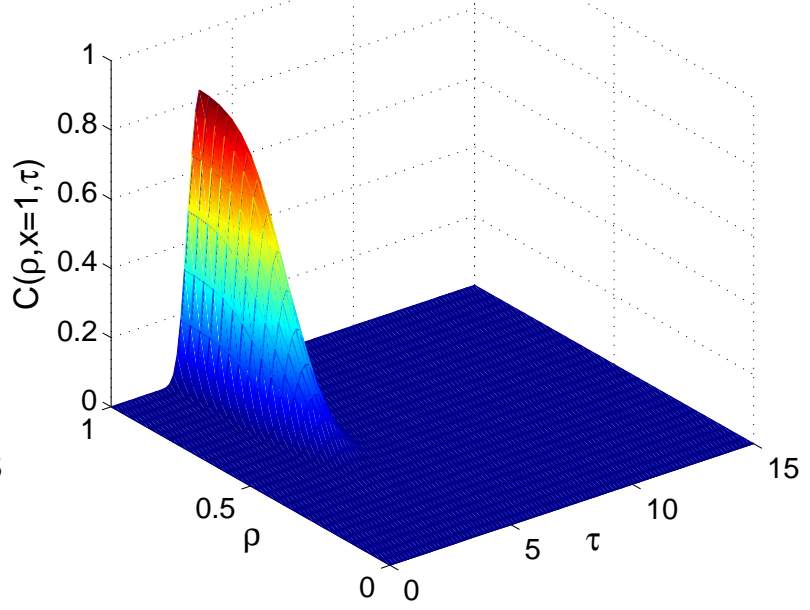

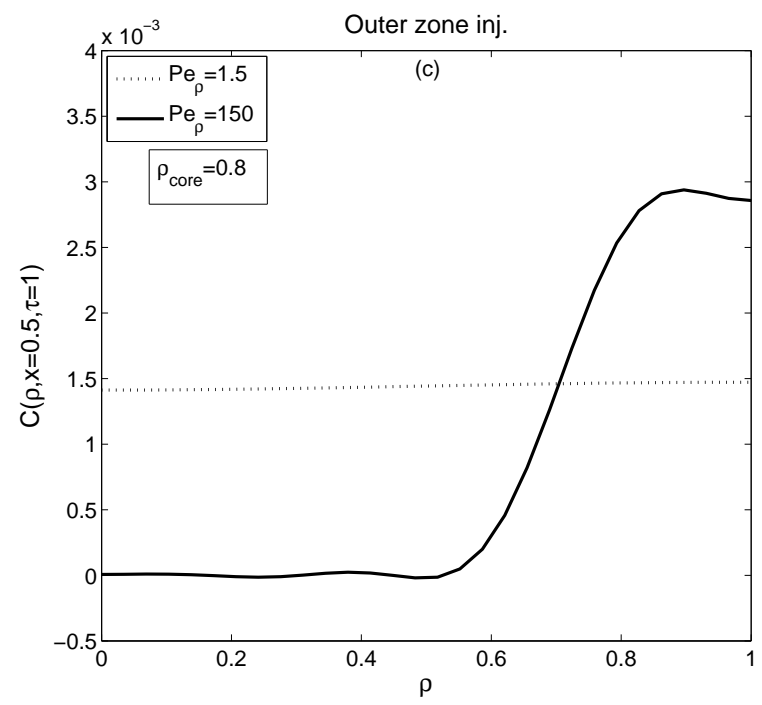

Figure 4: Linear isotherm and outer zone injection: Effects of the radial Peclet number $\left(P e_{\rho}\right)$ on the concentration profiles for $\rho_{\text {core }}=0.8$. The $1 \mathrm{D}$ plots in (c) are given at the centre of the column $x=0.5$. The results were obtained using the Danckwerts BC. 

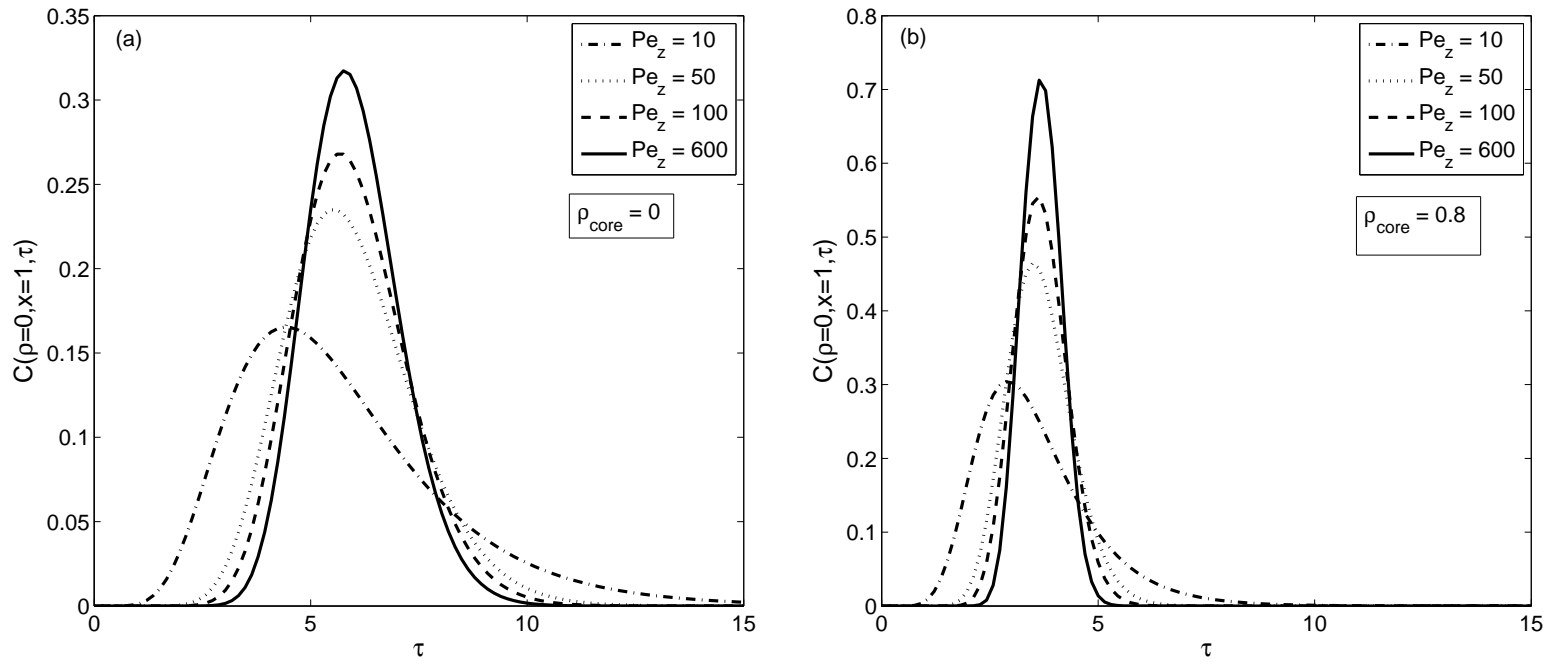

Figure 5: Linear isotherm and inner zone injection: 1D plots at $\rho=0$ and $x=1$ for showing the effect of axial Peclet number $\left(P e_{z}\right)$ on the elution profiles using Danckwerts BC. 

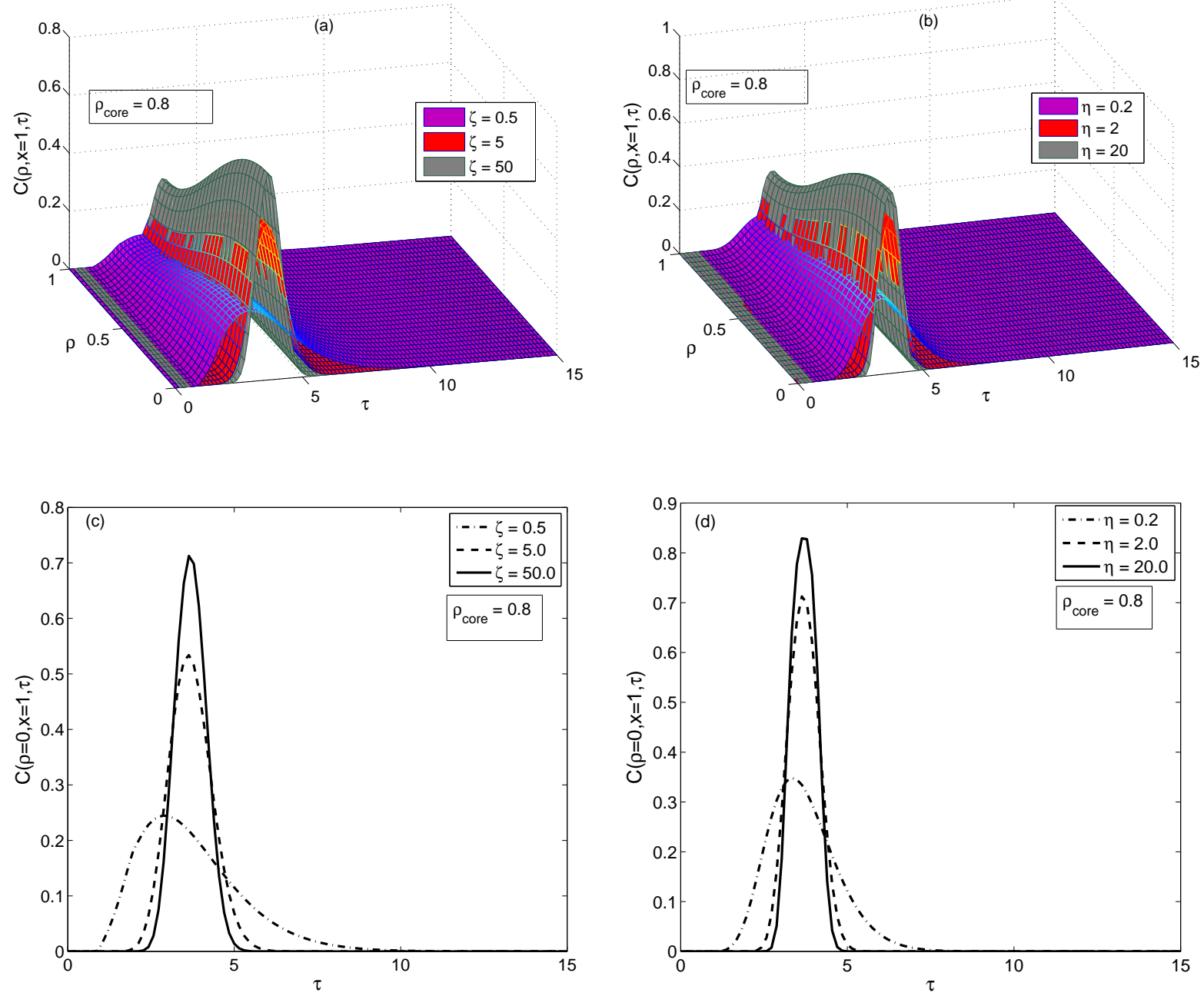

Figure 6: Linear isotherm and inner zone injection: Effects of mass transfer coefficients $\zeta$ and $\eta$ on the concentration profiles using Danckwerts BC. 

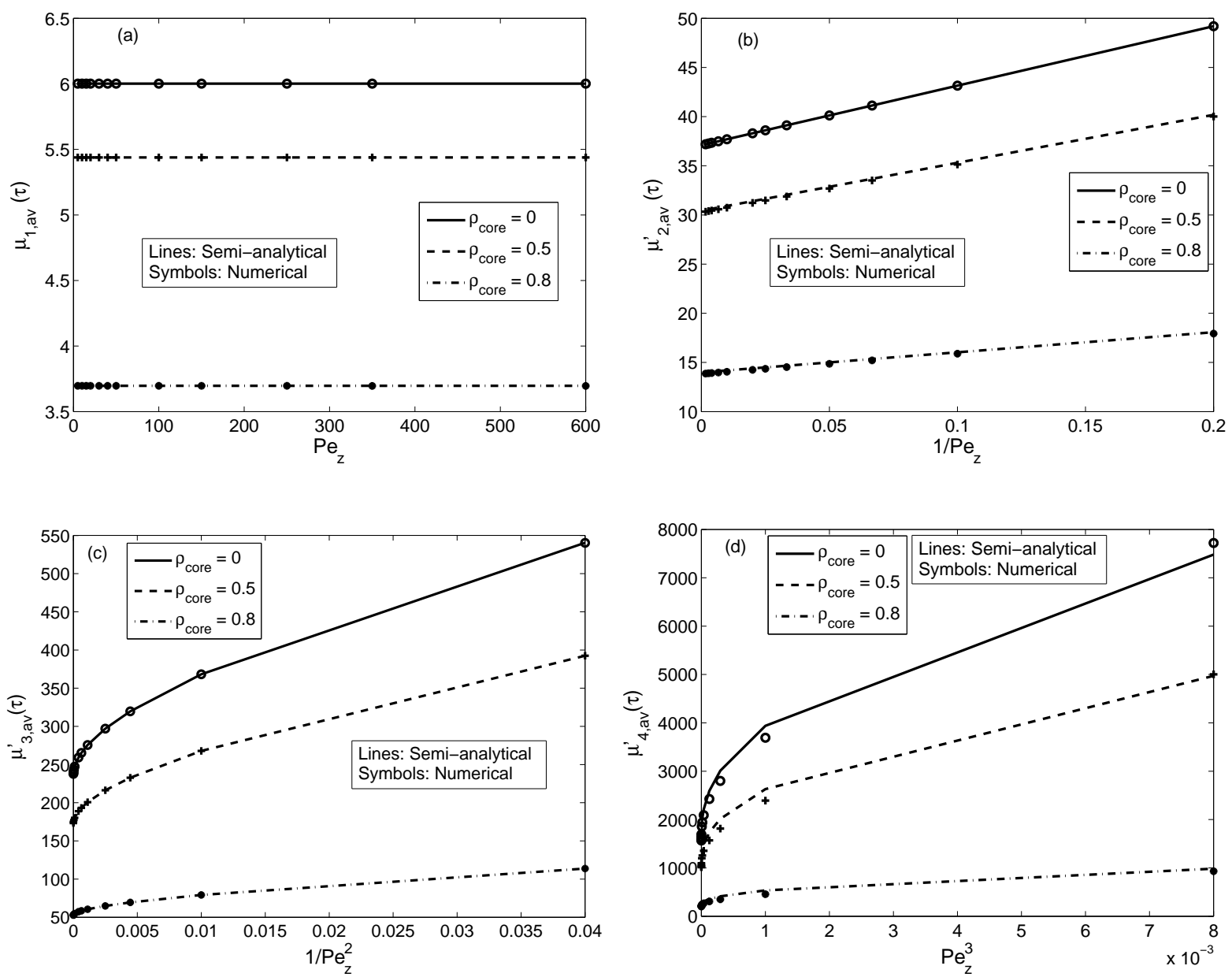

Figure 7: Linear isotherm and inner zone injection: Comparison of analytical and numerical moments for different values of $\rho_{\text {core }}$ using Dirichlet BC. 

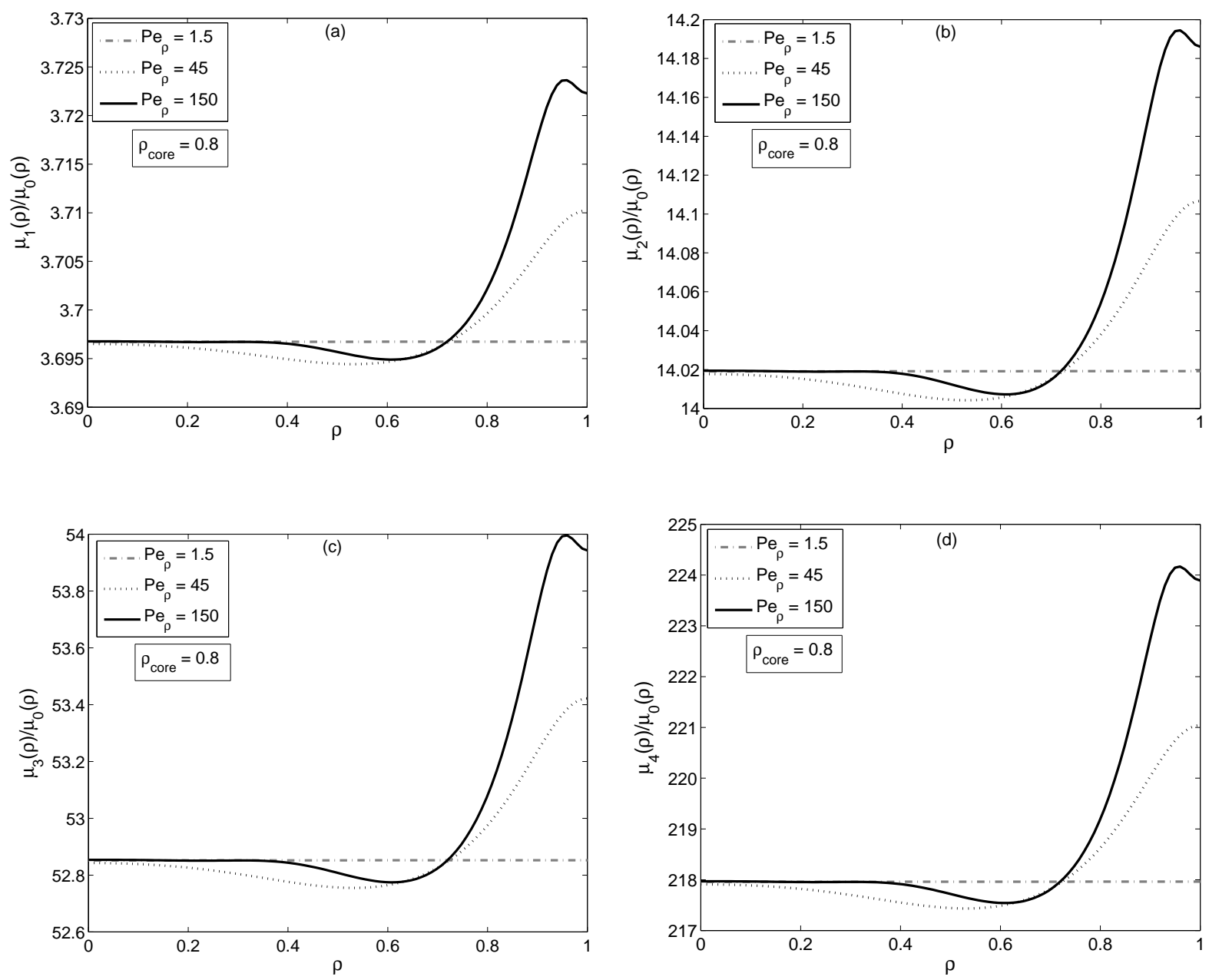

Figure 8: Linear isotherm and inner zone injection: Local moments showing the effect of radial peclet number $\left(P e_{\rho}\right)$ for $\rho_{\text {core }}=0.8$ using Dirichlet BC. 

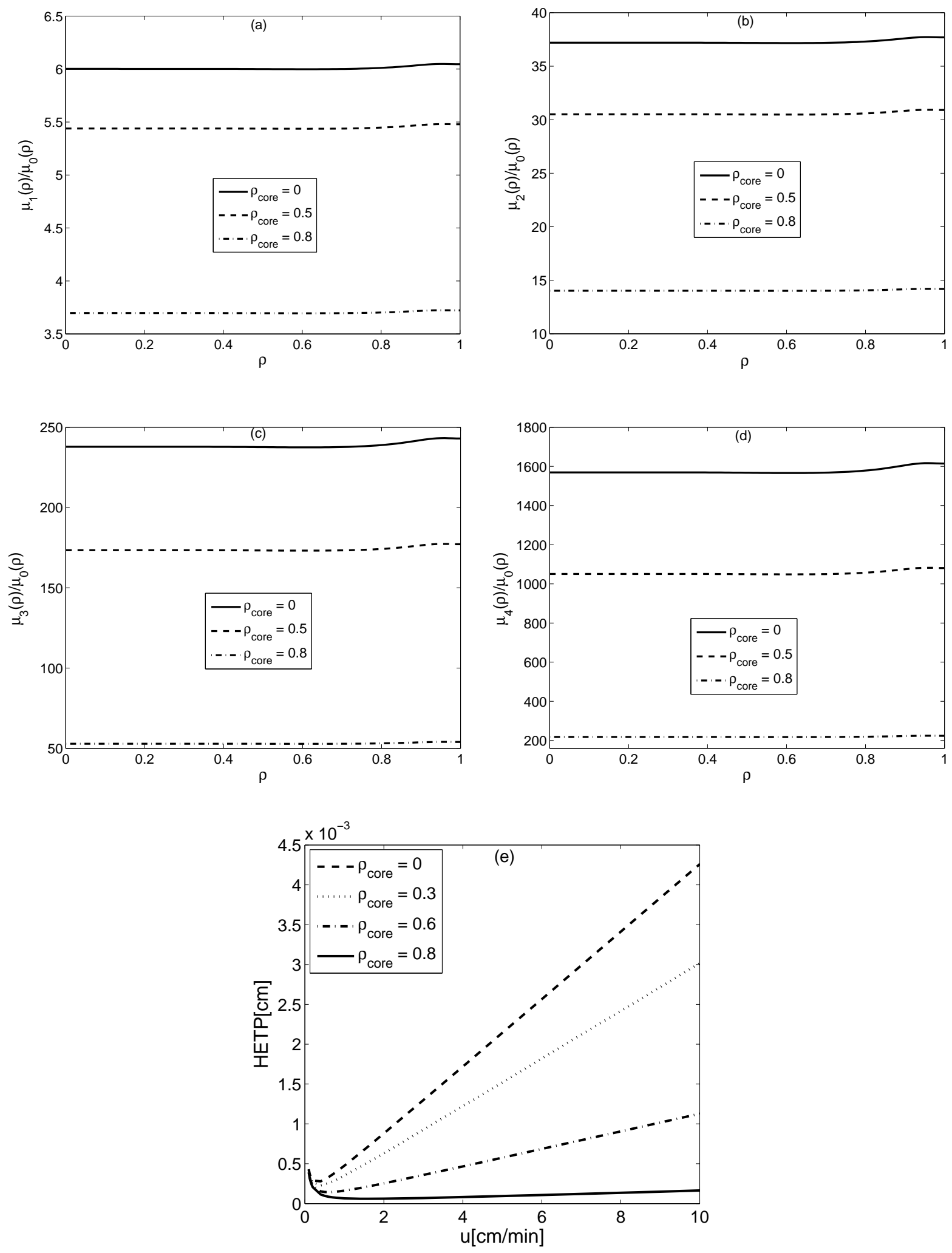

Figure 9: Linear isotherm: Plots (a)-(d) show the effects $\rho_{\text {core }}$ on local moments for $P e_{\rho}=150$ and $P e_{z}=600$. Plot (e) shows HETP curves for different values of $\rho_{\text {core }}$ over $u$ (c.f. Eq. (52)). The results were obtained using the Danckwerts BC. 

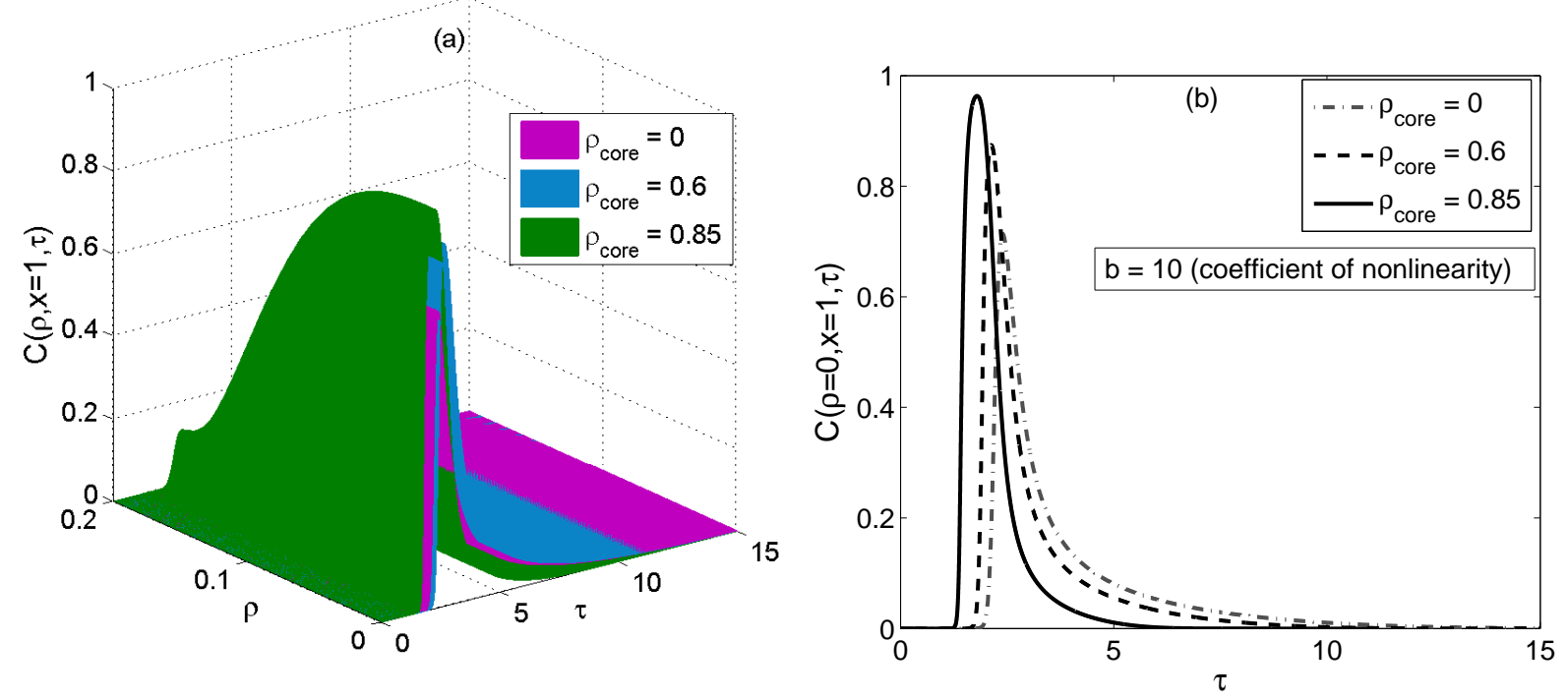

Figure 10: Nonlinear isotherm and inner zone injection: 3D and 1D plots of the nonlinear single-component elusion using Danckwerts BC. 

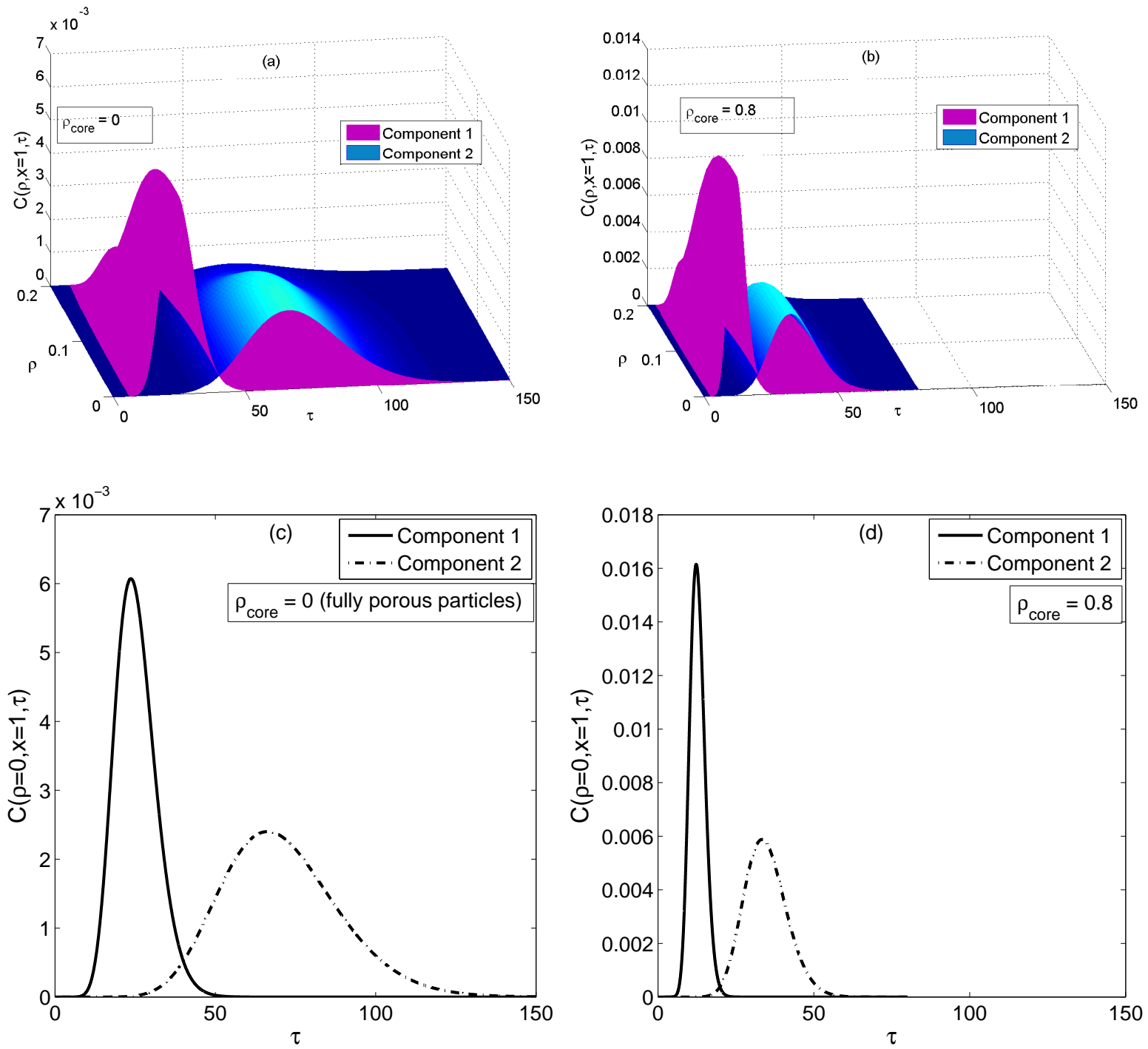

Figure 11: Nonlinear isotherm and inner zone injetion: 3D and 1D profiles for two-component mixture elusion using Danckwerts BC. Here, $\rho_{\text {core }}=0$ and $\rho_{\text {core }}=0.8$. 

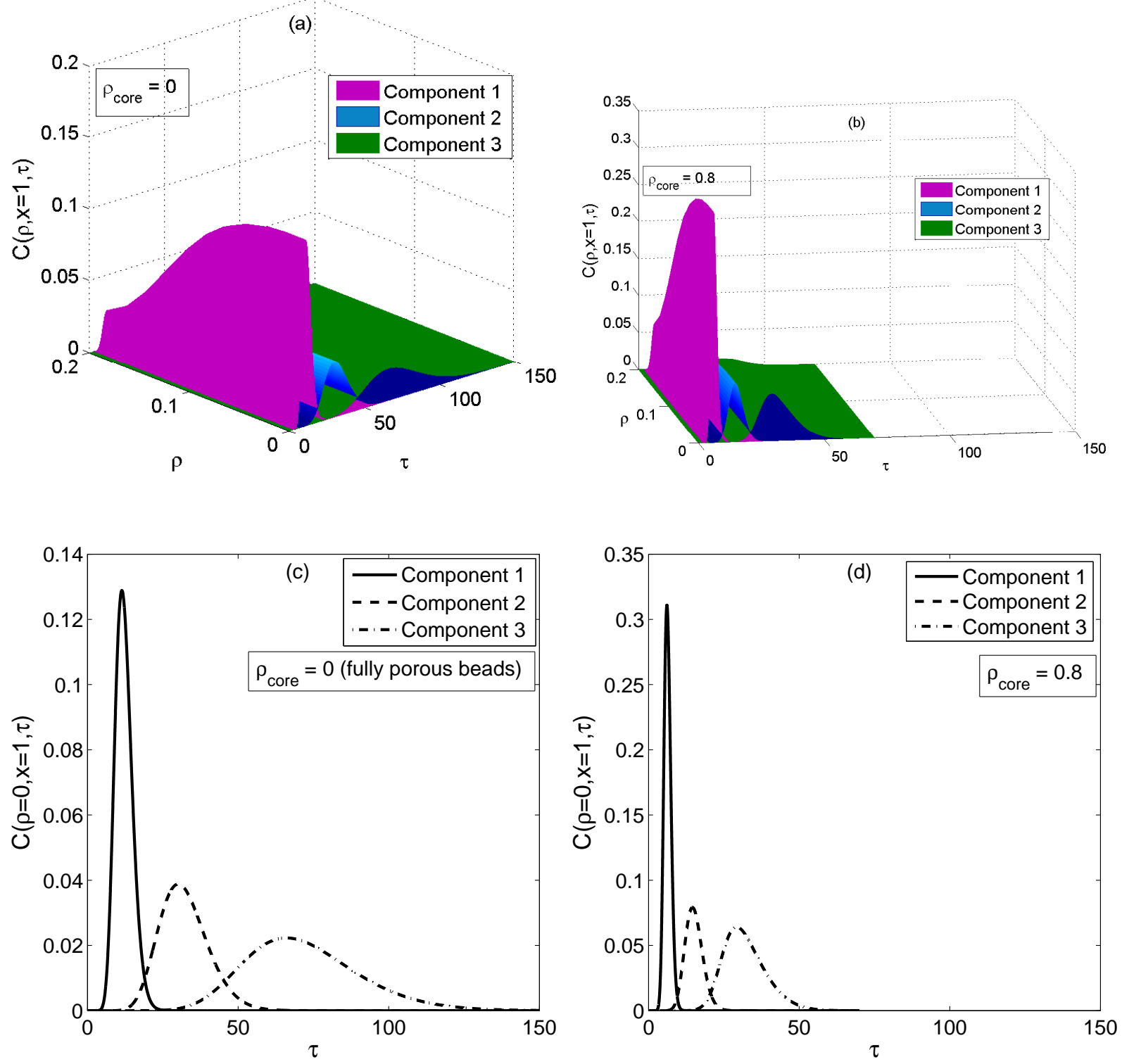

Figure 12: Nonlinear isotherm and inner zone injection: 3D and 1D profiles for three-component mixture elusion using Danckwerts BC. Here, $\rho_{\text {core }}=0$ and $\rho_{\text {core }}=0.8$. 

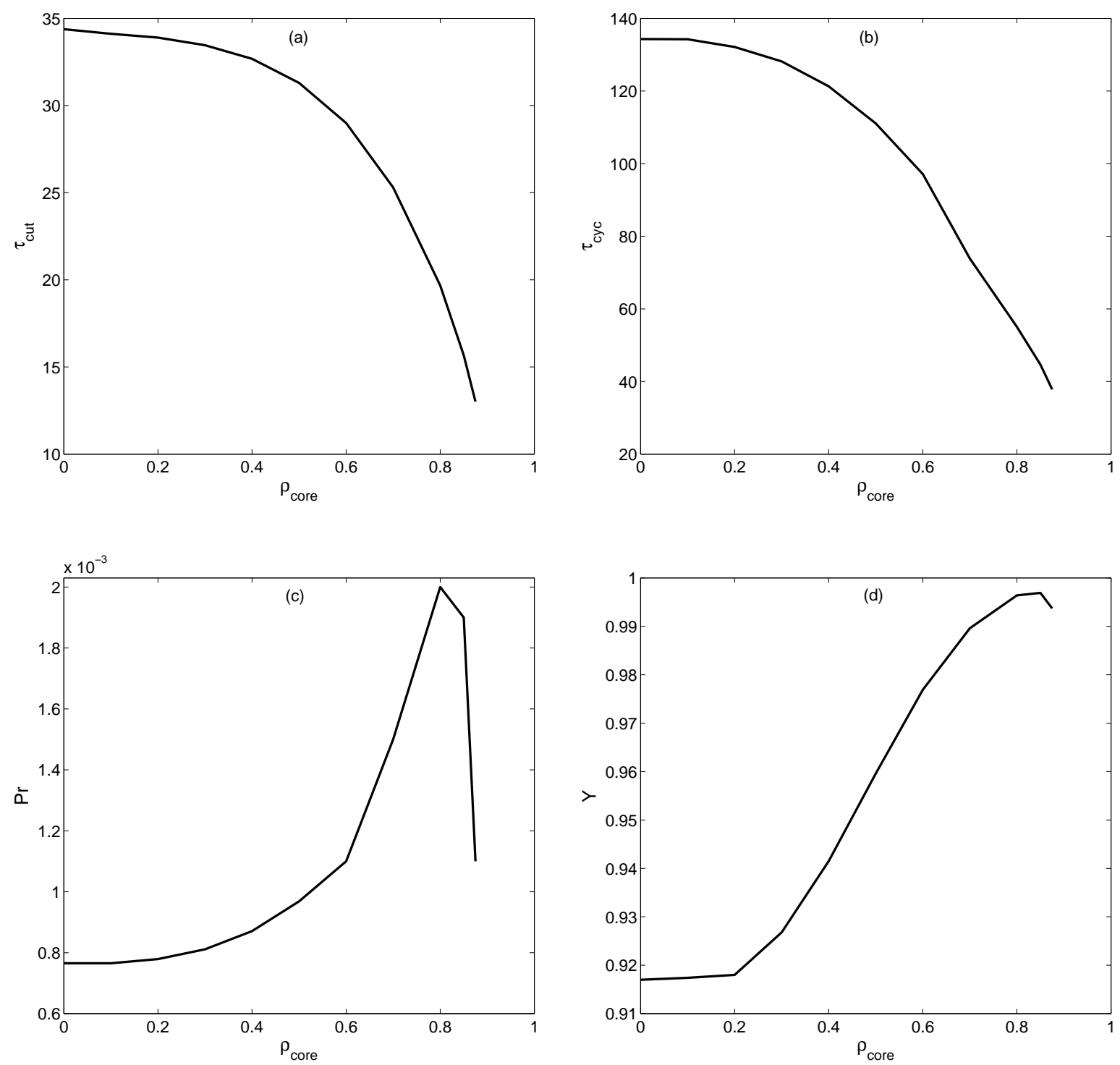

Figure 13: Nonlinear isotherm and two-component mixture: plots of (a) $\tau_{c u t}$, (b) $\tau_{c y c}$, (c) $\operatorname{Pr}$, and (d) $Y$ as functions of $\rho_{\text {core }}$ for fixed values of $c_{i n j, 1}=1=c_{i n j, 2}, \tau_{i n j}=0.1$ and $b_{1}=1=b_{2}$. The results were obtained using the Danckwerts BC. The values of other standard parameters are given in Table 1. 

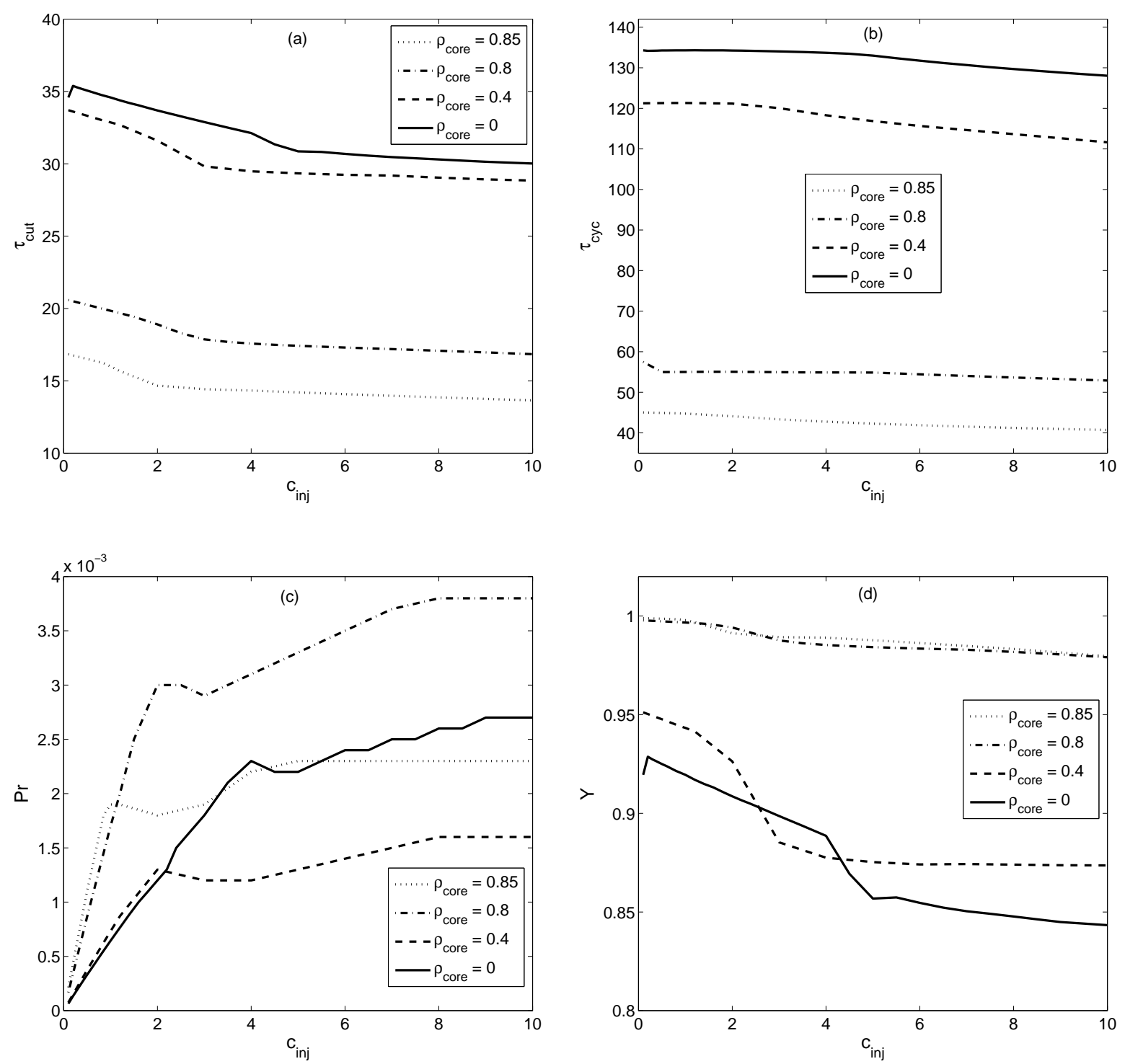

Figure 14: Nonlinear isotherm and two-component mixture: Plots of (a) $\tau_{c u t}$, (b) $\tau_{c y c}$, (c) $\operatorname{Pr}$, and (d) $Y$ as functions of $c_{i n j}$ for different values of $\rho_{\text {core }}$ with $c_{i n j, 1}=c_{i n j, 2}, \tau_{i n j}=0.1$ and $b_{1}=1=b_{2}$. The results were obtained using the Danckwerts BC. The values of other standard parameters are given in Table 1. 

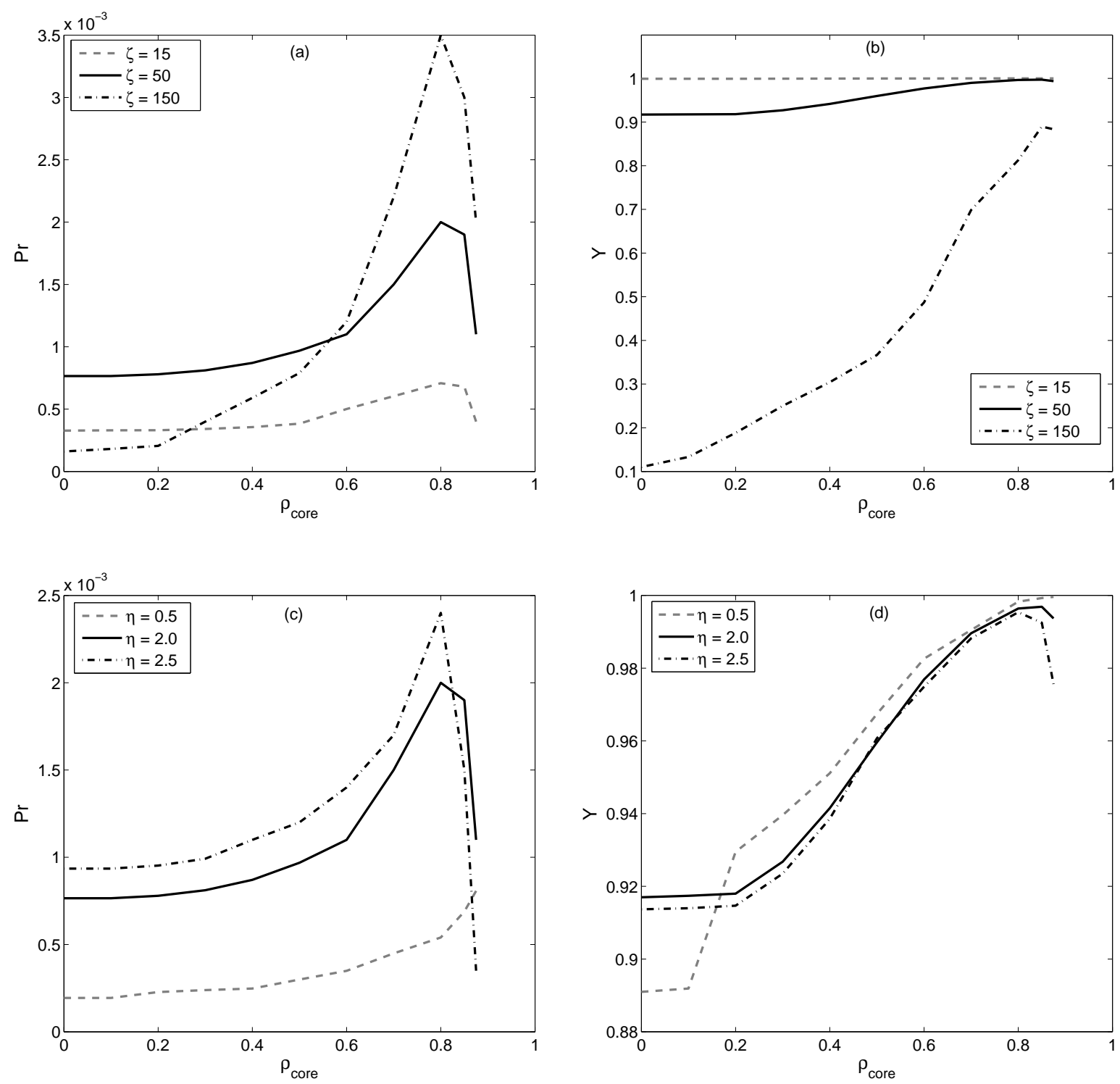

Figure 15: Nonlinear isotherm and two-component mixture: Plots (a), (b), (c) and (d) show the effects of $\zeta$ and $\eta$ on $\operatorname{Pr}$ and $Y$. Here, $c_{i n j, 1}=1.0=c_{i n j, 2}, \tau_{i n j}=0.1$ and $b_{1}=1=b_{2}$. The results were obtained using the Danckwerts BC. The values of other standard parameters are given in Table 1. 